\title{
MOTIVIC ZETA FUNCTIONS FOR CURVE SINGULARITIES
}

\author{
J. J. MOYANO-FERNÁNDEZ AND W. A. ZÚÑIGA-GALINDO
}

\begin{abstract}
Let $X$ be a complete, geometrically irreducible, singular, algebraic curve defined over a field of characteristic $p$ big enough. Given a local ring $\mathcal{O}_{P, X}$ at a rational singular point $P$ of $X$, we attached a universal zeta function which is a rational function and admits a functional equation if $\mathcal{O}_{P, X}$ is Gorenstein. This universal zeta function specializes to other known zeta functions and Poincaré series attached to singular points of algebraic curves. In particular, for the local ring attached to a complex analytic function in two variables, our universal zeta function specializes to the generalized Poincaré series introduced by Campillo, Delgado, and Gusein-Zade.
\end{abstract}

\section{$\S 1$. Introduction}

Let $X$ be a complete, geometrically irreducible, singular, algebraic curve defined over a finite field $\mathbb{F}_{q}$. In [29], the second author introduced a zeta function $Z(\mathrm{Ca}(X), T)$ associated to the effective Cartier divisors on $X$. Other types of zeta functions associated to singular curves over finite fields were introduced in [15], [16], [23], [24], and [30]. The zeta function $Z(\mathrm{Ca}(X), T)$ admits an Euler product with nontrivial factors at the singular points of $X$. If $P$ is a rational singular point of $X$, then the local factor $Z_{\mathrm{Ca}(X)}\left(T, q, \mathcal{O}_{P, X}\right)$ at $P$ is a rational function of $T$ depending on $q$ and the completion $\widehat{\mathcal{O}}_{P, X}$ of the local ring $\mathcal{O}_{P, X}$ of $X$ at $P$. If the residue field of $\widehat{\mathcal{O}}_{P, X}$ is not too small, then $Z_{\mathrm{Ca}(X)}\left(T, q, \mathcal{O}_{P, X}\right)$ depends only on the semigroup of $\widehat{\mathcal{O}}_{P, X}$ (see $\left[29\right.$, Theorem 5.5]). Thus, if $\widehat{\mathcal{O}}_{P, X} \cong \mathbb{F}_{q} \llbracket x, y \rrbracket /(f(x, y))$,

Received January 10, 2009. Revised September 1, 2009. Accepted September 10, 2009. 2000 Mathematics Subject Classification. Primary 14H20, 14G10; Secondary 32S40, $11 \mathrm{~S} 40$.

The first author was partially supported by Ministerio de Educación y Ciencia grant MTM2007-64704, by Junta de Castilla y León grant VA065A07, and by a grant from Deutscher Akademischer Austausch Dienst-La Caixa 2007.

(C) 2010 by The Editorial Board of the Nagoya Mathematical Journal 
then $Z_{\mathrm{Ca}(X)}\left(T, q, \mathcal{O}_{P, X}\right)$ becomes a complete invariant of the equisingularity class of the algebroid curve $\widehat{\mathcal{O}}_{P, X}$ (see [4], [26], [28]). Motivated by [12], in [31] the second author computed several examples showing that $\lim _{q \rightarrow 1} Z_{\mathrm{Ca}(X)}\left(T, q, \mathcal{O}_{P, X}\right)$ equals the zeta function of the monodromy of the (complexification) of $f$ at the origin (see [1] and the examples in Section 9). In this article we study this phenomenon.

By using motivic integration in the spirit of Campillo, Delgado, and Gusein-Zade, we attach to a local ring $\mathcal{O}_{P, X}$ of an algebraic curve $X$ a "universal zeta function" (see Definition 5 , Theorem 1, Definition 8). This zeta function specializes to $Z_{\mathrm{Ca}(X)}\left(T, q, \mathcal{O}_{P, X}\right)$ (see Lemma 7 and Theorem 3 ). We also establish that $\lim _{q \rightarrow 1} Z_{\mathrm{Ca}(X)}\left(T, q, \mathcal{O}_{P, X}\right)$ equals a zeta function of the monodromy of a reduced complex mapping in two variables at the origin (see Theorem 3). A key ingredient is a result of Campillo, Delgado, and Gusein-Zade relating the Poincaré series attached to complex analytic functions in two variables and the zeta function of the monodromy (see [4] and Theorem 2). From the point of view of the work of Campillo, Delgado, and Gusein-Zade, this article deals with Poincaré series attached to local rings $\mathcal{O}_{P, X}$ when the ground field is big enough (see Lemma 4). In particular, for the local ring attached to a complex analytic function in two variables, our universal zeta function specializes to the generalized Poincaré series introduced in [7], and then a relation with the Alexander polynomial holds as a consequence of [6]. We also obtain explicit formulas that give precise information about the degree of the numerators of such Poincaré series and functional equations (see Theorem 4, Corollary 5, and Remark 13). Our results suggest that the factor $Z_{\mathrm{Ca}(X)}\left(T, q, \mathcal{O}_{P, X}\right)$ is the "monodromy zeta function of $\mathcal{O}_{P, X}$." In order to understand this, we believe that a cohomological theory for the universal zeta functions should be developed.

Finally, we want to comment that the connections between zeta functions introduced here and the motivic zeta functions of Kapranov [17] and Baldassarri, Deninger, and Naumann [3] are unknown. However, we believe that the zeta functions introduced here are factors of motivic zeta functions of Baldassarri-Deninger-Naumann type for singular curves. In a forthcoming paper we plan to address this connection. For a general discussion about motivic zeta functions for curves, the reader may consult [2, and the references therein] and [14]. 


\section{$\S 2$. The semigroup of values of a curve singularity}

Let $X$ be a complete, geometrically irreducible, algebraic curve defined over a field $k$, with function field $K / k$. Let $\widetilde{X}$ be the normalization of $X$ over $k$, and let $\pi: \widetilde{X} \rightarrow X$ be the normalization map. Let $P \in X$ be a closed point of $X$, and let $O_{P}=O_{P, X}$ be the local ring of $X$ at $P$. Let $Q_{1}, \ldots, Q_{d}$ be the points of $\widetilde{X}$ lying over $P$, that is, $\pi^{-1}(P)=\left\{Q_{1}, \ldots, Q_{d}\right\}$, and let $O_{Q_{1}}, \ldots, O_{Q_{d}}$ be the corresponding local rings at these points. Since the function fields of $\widetilde{X}$ and $X$ are the same, and $\widetilde{X}$ is a nonsingular curve, the local rings $O_{Q_{1}}, \ldots, O_{Q_{d}}$ are valuation rings of $K / k$ over $O_{P}$. The integral closure of $O_{P}$ in $K / k$ is $\mathbb{O}_{P}=O_{Q_{1}} \cap \cdots \cap O_{Q_{d}}$.

Let $\widehat{\mathbb{O}}_{P}$ be the completion of $\mathbb{O}_{P}$ with respect to its Jacobson ideal, and let $\widehat{O}_{P}$ (resp., $\widehat{O}_{Q_{i}}$ ) be, for $i=1, \ldots, d$, the completion of $O_{P}$ (resp., of $O_{Q_{i}}$ ) for $i=1, \ldots, d$, with respect to the topology induced by their maximal ideals. We denote by $B_{P}^{(j)}, j=1, \ldots, d$, the minimal primes of $\widehat{O}_{P}$. Then we have the diagram

$$
\begin{aligned}
& \widehat{\mathbb{O}}_{P} \cong \widehat{O}_{Q_{1}} \times \cdots \times \widehat{O}_{Q_{d}} \\
& \uparrow \widehat{\uparrow}_{P} \underset{\widehat{O}_{P}}{\underline{O}} \widehat{O}_{B_{P}^{(1)}} \times \cdots \times \widehat{O}_{B_{P}^{(d)}},
\end{aligned}
$$

where $\underline{\varphi}$ is the diagonal morphism. Since $\widehat{O}_{P}$ is a reduced ring (see $[20$, Theorem 1], [27, proof of Satz 3.6]), $\varphi$ is one to one. Thus we have a bijective correspondence between the $\widehat{O}_{Q_{i}}$ and $\widehat{O}_{B_{P}^{(i)}}$. We call the rings $\widehat{O}_{B_{P}^{(i)}}$ the branches of $\widehat{O}_{P}$. By the Cohen structure theorem for complete regular local rings, each $\widehat{O}_{Q_{i}}$ is isomorphic to $k_{i} \llbracket t_{i} \rrbracket, i=1, \ldots, d$, where $k_{i}$ is the residue field of $\widehat{O}_{Q_{i}}$.

We will say that $\widehat{O}_{P}$ is totally rational if all rings $\widehat{O}_{Q_{i}}$, for $i=1, \ldots, d$, have $k$ as a residue field.

From now on we assume that $\widehat{O}_{P}$ is a totally rational ring, and we identify $\widehat{O}_{P}$ with $\varphi\left(\widehat{O}_{P}\right)$. Let $v_{i}$ denote the valuation associated with $\widehat{O}_{Q_{i}}$, $i=1, \ldots, d$. By using these valuations, we define $\underline{v}(\underline{z})=\left(v_{1}\left(z_{1}\right), \ldots, v_{d}\left(z_{d}\right)\right)$ for any nonzero divisor $\underline{z}=\left(z_{1}, \ldots, z_{d}\right) \in \widehat{\mathbb{O}}_{P}$.

The semigroup $S$ of values of $\widehat{O}_{P}$ consists of all the elements of the form $\underline{v}(\underline{z})=\left(v_{1}\left(z_{1}\right), \ldots, v_{d}\left(z_{d}\right)\right) \in \mathbb{N}^{d}$ for all the nonzero divisors $\underline{z} \in \widehat{O}_{P}$. Observe that, by definition, the semigroup of $\widehat{O}_{P}$ coincides with the semigroup of values of $O_{P}$. 
We set $\underline{z}=\underline{t} \underline{\underline{n}} \underline{\mu}:=\left(t_{1}^{n_{1}}, \ldots, t_{d}^{n_{d}}\right)\left(\mu_{1}, \ldots, \mu_{d}\right)=\left(t_{1}^{n_{1}} \mu_{1}, \ldots, t_{d}^{n_{d}} \mu_{d}\right)$, with $\underline{\mu}=$ $\left(\mu_{1}, \ldots, \mu_{d}\right) \in \widehat{\mathbb{O}}_{P}^{\times}$. With this notation, the ideal generated by a nonzero divisor of $\widehat{\mathbb{O}}_{P}$ has the form $\underline{t} \underline{\underline{n}} \widehat{\mathbb{O}}_{P}$, for some $\underline{n} \in \mathbb{N}^{d}$.

We set $\underline{1}:=(1, \ldots, 1) \in \mathbb{N}^{d}$, and for $\underline{n}=\left(n_{1}, \ldots, n_{d}\right) \in \mathbb{N}^{d},\|\underline{n}\|:=$ $n_{1}+\cdots+n_{d}$. We introduce a partial order in $\mathbb{N}^{d}$, the product order, by taking $\underline{n} \geqslant \underline{m}$, if $n_{i} \geqslant m_{i}$ for $i=1, \ldots, d$.

There exists $\underline{c}_{P}=\left(c_{1}, \ldots, c_{d}\right) \in \mathbb{N}^{d}$ minimal for the product order such that $\underline{c}_{P}+\mathbb{N}^{d} \subseteq S$. This element is called the conductor of $S$. The conductor ideal $\widehat{F}_{P}$ of $\widehat{O}_{P}$ is $\underline{t}_{P} \widehat{\mathbb{O}}_{P}$. This is the largest common ideal of $\widehat{O}_{P}$ and $\widehat{\mathbb{O}}_{P}$. The singularity degree $\delta_{P}$ of $\widehat{O}_{P}$ is defined as $\delta_{P}:=\operatorname{dim}_{k} \widehat{\mathbb{O}}_{P} / \widehat{O}_{P}<\infty$ (see, e.g., [22, Chapter IV]). If $\widehat{O}_{P}$ is a Gorenstein ring, the singularity degree is related to the conductor by the equality $\left\|\underline{c}_{P}\right\|=2 \delta_{P}$ (see, e.g., [22, Chapter IV]). By using the facts that $\widehat{O}_{P} / \widehat{F}_{P}$ is a $k$-subalgebra of $\widehat{\mathbb{O}}_{P} / \widehat{F}_{P}$ of codimension $\delta_{P}$, that $\widehat{\mathbb{O}}_{P} / \widehat{F}_{P}$ is a finite-dimensional $k$-algebra, and that $\widehat{F}_{P}$ is a common ideal of $\widehat{O}_{P}$ and $\widehat{\mathbb{O}}_{P}$, we have

$$
\widehat{O}_{P}=\left\{\left(\sum_{i=0}^{\infty} a_{i, 1} t_{1}^{i}, \ldots, \sum_{i=0}^{\infty} a_{i, d} t_{d}^{i}\right) \in \widehat{\mathbb{O}}_{P} \mid \Delta=0\right\},
$$

where $\Delta=0$ denotes a homogeneous system of linear equations involving only a finite number of the $a_{i, j}$. Indeed,

$$
c_{m}=1+\max \left\{i \mid a_{i, m} \text { appears in } \Delta=0\right\},
$$

for $m=1, \ldots, d$ (see examples in Section 9 ). Note that, as a consequence of the definition of $\underline{\varphi}$, the relations $a_{0,1}=a_{0,2}=\cdots=a_{0, d}$ hold.

REMARK 1. (Conventions and notation) (1) From now on we will use " $X$ is an algebraic curve over $k$," to mean that $X$ is a complete, geometrically irreducible, algebraic curve over $k$.

(2) To simplify the notation, we drop the index $P$, and we denote $\widehat{O}_{P}$ by $\mathcal{O}, \widehat{F}_{P}$ by $\mathcal{F}$, and $\widehat{\mathbb{O}}_{P}$ by $\widetilde{\mathcal{O}}=k \llbracket t_{1} \rrbracket \times \cdots \times k \| t_{d} \rrbracket ; \mathcal{O}$ is a $k$-vector space of finite codimension in $\widetilde{\mathcal{O}}$ with presentation $(2.1)$. We also drop the index $P$ from $\underline{c}_{P}$ and $\delta_{P}$.

REMARK 2. Let $(X, 0) \subset\left(\mathbb{C}^{2}, 0\right)$ be a germ of reduced plane curve given by $f=0$ for $f \in \mathcal{O}_{\left(\mathbb{C}^{2}, 0\right)}$, and let $X=\bigcup_{i=1}^{d} X_{i}$ with $d \geqslant 1$ be its decomposition into irreducible components (or branches) corresponding to $f=\prod_{i=1}^{d} f_{i}$. Let $\mathcal{O}:=\mathcal{O}_{(X, 0)}=\mathcal{O}_{\left(\mathbb{C}^{2}, 0\right)} /(f)$ be the ring of germs of analytic functions 
on $X$. Let $\varphi_{i}:(\mathbb{C}, 0) \rightarrow\left(\mathbb{C}^{2}, 0\right)$ be a parametrization of $X_{i}$; that is, $\varphi_{i}$ is an isomorphism between $X_{i}$ and $\mathbb{C}$ outside of the origin, for $i=1, \ldots, d$. Let $S(\mathcal{O}):=S(f)$ denote the semigroup of $\mathcal{O}$ defined by using the parametrizations $\varphi_{i}$. (For further details, see, e.g., [9].)

\section{$\S 3$. Integration with respect to the generalized Euler characteristic}

We denote by $\operatorname{Var}_{k}$ the category of $k$-algebraic varieties, and by $K_{0}\left(\operatorname{Var}_{k}\right)$ the corresponding Grothendieck ring, which is the ring generated by symbols $[V]$, for $V$ an algebraic variety, with the relations $[V]=[W]$ if $V$ is isomorphic to $W,[V]=[V \backslash Z]+[Z]$ if $Z$ is closed in $V$, and $[V \times W]=[V][W]$. We denote by $\mathbf{1}:=\left[\right.$ point], $\mathbb{L}:=\left[\mathbb{A}_{k}^{1}\right]$, and $\mathcal{M}_{k}:=K_{0}\left(\operatorname{Var}_{k}\right)\left[\mathbb{L}^{-1}\right]$ the ring obtained by localization with respect to the multiplicative set generated by $\mathbb{L}$.

We define the set of $\underline{n}$-jets $J_{\widetilde{\mathcal{O}}}^{n}$ of the local ring $\widetilde{\mathcal{O}}$ as $J_{\widetilde{\mathcal{O}}}=\widetilde{\mathcal{O}} / t^{\underline{n}}+\underline{1} \widetilde{\mathcal{O}} \cong$ $k^{\| \underline{n}+1} \|$. The canonical projection $\widetilde{\mathcal{O}} \rightarrow \widetilde{\mathcal{O}} / \underline{\underline{n}} \underline{\underline{n}}+\underline{\widetilde{\mathcal{O}}}$ is denoted by $\pi_{\underline{n}}$.

Definition 1. A subset $X \subseteq \widetilde{\mathcal{O}}=k \llbracket t_{1} \rrbracket \times \cdots \times k \llbracket t_{d} \rrbracket$ is said to be cylindric if $X=\pi_{\underline{n}}^{-1}(Y)$ for a constructible subset $Y$ of $J \frac{n}{\widetilde{\mathcal{O}}}$.

We note that $\mathcal{O}$ and $\mathcal{O}^{\times}$(the group of units of $\mathcal{O}$ ) are cylindric subsets of $\widetilde{\mathcal{O}}($ see $(2.1))$.

REMARK 3. Any constructible subset $Y$ of $J \frac{n}{\widetilde{\mathcal{O}}}$ is defined by a condition that can be expressed as a finite Boolean combination of conditions of the form

$$
\left\{\begin{array}{l}
p_{i}\left(x_{0}, \ldots, x_{m-1}\right)=0, \quad i \in I \\
q\left(x_{0}, \ldots, x_{m-1}\right) \neq 0
\end{array}\right.
$$

where $m=\|\underline{n}+\underline{1}\|, p_{i}\left(x_{0}, \ldots, x_{m-1}\right)$ and $q\left(x_{0}, \ldots, x_{m-1}\right)$ are polynomials in $k\left[x_{0}, \ldots, x_{m-1}\right]$, and $I$ is a finite subset independent of $m$. We call such a condition constructible in $J \frac{n}{\widetilde{\mathcal{O}}}$. Definition 1 means that the condition for a function $\underline{z} \in \widetilde{\mathcal{O}}$ to belong to the set $X$ is a constructible condition on the $\underline{n}$-jet $\pi_{\underline{n}}(\underline{z})$ of $\underline{z}$.

We present now the notion of integral with respect to the generalized Euler characteristic introduced by Campillo, Delgado, and Gusein-Zade in [7] for the complex case and by Delgado and Moyano in [11] for more general contexts. 
Definition 2. The generalized Euler characteristic (or motivic measure) of a cylindric subset $X \subseteq \widetilde{\mathcal{O}}, X=\pi_{\underline{n}}^{-1}(Y)$, with $Y \subseteq J \frac{n}{\widetilde{\mathcal{O}}}$ constructible, is $\chi_{g}(X):=[Y] \mathbb{L}^{-\|\underline{n}+\underline{1}\|} \in \mathcal{M}_{k}$.

The generalized Euler characteristic $\chi_{g}(X)$ is well defined since, if $X=$ $\pi_{\underline{m}}^{-1}\left(Y^{\prime}\right), Y^{\prime} \subseteq J \frac{m}{\widetilde{\mathcal{O}}}$, and $\underline{n} \geqslant \underline{m}$, then $Y$ is a locally trivial fibration over $Y^{\prime}$ with fiber $k^{r}$, where $r=\|\underline{n}+\underline{1}\|-\|\underline{m}+\underline{1}\|$.

Definition 3. Let $(G,+, 0)$ be an abelian group, and let $X$ be a cylindric subset of $\widetilde{\mathcal{O}}$. A function $\phi: \widetilde{\mathcal{O}} \rightarrow G$ is called cylindric if it has countably many values, and for each $a \in G, a \neq 0$, the set $\phi^{-1}(a)$ is cylindric. As in [13] and [7], we define

$$
\int_{X} \phi d \chi_{g}=\sum_{\substack{a \in G \\ a \neq 0}} \chi_{g}\left(X \cap \phi^{-1}(a)\right) \otimes a
$$

if the sum has sense in $G \otimes_{\mathbb{Z}} \mathcal{M}_{k}$. In such a case, the function $\phi$ is said to be integrable over $X$.

Now we give the projective versions of the above definitions which we will use later on. For a $k$-vector space $L$ (finite or infinite dimensional), let $\mathbb{P} L=(L \backslash\{0\}) / k^{\times}$be its projectivization, and let $\mathbb{P}^{\times} L$ be the disjoint union of $\mathbb{P} L$ with a point $\left(\mathbb{P}^{\times} L\right.$ can be identified with $\left.L / k^{\times}\right)$. The natural map $\mathbb{P} \widetilde{\mathcal{O}} \rightarrow \mathbb{P}^{\times} J \frac{n}{\widetilde{\mathcal{O}}}$ is also denoted by $\pi_{\underline{n}}$.

Definition 4. A subset $X \subseteq \mathbb{P} \widetilde{\mathcal{O}}$ is said to be cylindric if $X=\pi_{n}^{-1}(Y)$ for a constructible subset $Y$ of $\mathbb{P} J \frac{n}{\widetilde{\mathcal{O}}} \subset \mathbb{P}^{\times} J \frac{n}{\widetilde{\mathcal{O}}}$. The generalized Euler characteristic $\chi_{g}(X)$ of $X$ is $\chi_{g}(X):=[Y] \mathbb{L}^{-\|\underline{n}+\underline{1}\|} \in \mathcal{M}_{k}$.

A function $\phi: \mathbb{P} \widetilde{\mathcal{O}} \rightarrow G$ is called cylindric if it satisfies the conditions in Definition 3. The notion of integration over a cylindric subset of $\mathbb{P} \widetilde{\mathcal{O}}$ with respect to $d \chi_{g}$ follows the pattern of Definition 3 .

REMARK 4 . Let $V$ be a cylindric subset and a $k$-vector subspace of $\widetilde{\mathcal{O}}$. Let $\pi$ be the factorization map $\widetilde{\mathcal{O}} \backslash\{0\} \rightarrow \mathbb{P} \widetilde{\mathcal{O}}$, let $\Omega: \mathbb{P} \widetilde{\mathcal{O}} \rightarrow G$ be a cylindric function integrable over $\mathbb{P} V$, and define $\bar{\Omega}:=\Omega \circ \pi: \widetilde{\mathcal{O}} \backslash\{0\} \rightarrow G$. Then $\bar{\Omega}$ is a cylindric function integrable over $V$, and

$$
\int_{V} \bar{\Omega} d \chi_{g}=(\mathbb{L}-1) \int_{\mathbb{P} V} \Omega d \chi_{g} .
$$


The identity follows from the fact that

$$
\chi_{g}\left(\bar{\Omega}^{-1}(a) \cap V\right)=(\mathbb{L}-1) \chi_{g}\left(\Omega^{-1}(a) \cap \mathbb{P} V\right), \quad \text { for } a \in G, a \neq 0 .
$$

\section{$\S 4$. The structure of the algebraic group $\mathcal{J}$}

In this section $k$ is a field of characteristic zero. The quotient group $\widetilde{\mathcal{O}}^{\times} /(\underline{1}+\mathcal{F})$ admits a polynomial system of representatives $\left(g_{1}, \ldots, g_{i}\right.$, $\left.\ldots, g_{d}\right)$, where $g_{i}=\sum_{j=0}^{c_{i}-1} a_{j, i} t_{i}^{j}$, with $a_{0, i} \in k^{\times}$, and $\underline{c}=\left(c_{1}, \ldots, c_{d}\right)$ is the conductor of $S$. Thus $\widetilde{\mathcal{O}}^{\times} /(\underline{1}+\mathcal{F})$ can be considered as an open subset of the affine space of dimension $\|\underline{c}\|$; this algebraic structure is compatible with the group structure of $\widetilde{\mathcal{O}}^{\times} /(\underline{1}+\mathcal{F})$ (see [22, Chapter V, Section 14]). Furthermore,

$$
\widetilde{\mathcal{O}}^{\times} /(\underline{1}+\mathcal{F}) \cong\left(G_{m}\right)^{d} \times\left(G_{a}\right)^{\|\underline{c}\|-d},
$$

as algebraic groups, where $G_{m}=\left(k^{\times}, \cdot\right)$ and $G_{a}=(k,+)$ (see [22, Chapter V, Section 14]). By the discussion in Section 2, the group $\mathcal{O}^{\times} /(\underline{1}+\mathcal{F})$ is an algebraic subgroup of $\widetilde{\mathcal{O}}^{\times} /(\underline{1}+\mathcal{F})$.

We note that every equivalence class in $\pi_{\underline{c}-1}\left(\mathcal{O}^{\times}\right)$has a polynomial representative; then $\pi_{\underline{c}-1}\left(\mathcal{O}^{\times}\right)$can be considered an open subset of an affine space, and the multiplication in $\mathcal{O}^{\times}$induces a structure of algebraic group in $\pi_{\underline{c}-\underline{1}}\left(\mathcal{O}^{\times}\right)$. In addition, $\pi_{\underline{c}-1}\left(\widetilde{\mathcal{O}}^{\times}\right) \cong \widetilde{\mathcal{O}}^{\times} /(\underline{1}+\mathcal{F})$, as algebraic groups.

We set $\mathcal{J}:=\widetilde{\mathcal{O}}^{\times} / \mathcal{O}^{\times}$. Every equivalence class has a polynomial representative that can be identified with an element of $J \frac{c-1}{\widetilde{\mathcal{O}}}$. Each equivalence class depends on $\delta$ coefficients $a_{i, j}$ (see $(2.1)$ ); $d-1$ of them run over $k^{\times}$, and the others run over $k$. This set of polynomial representatives with the operation induced by the multiplication in $\widetilde{\mathcal{O}}^{\times}$is a $k$-algebraic group of dimension $\delta$; more precisely, $\mathcal{J} \cong\left(G_{m}\right)^{d-1} \times\left(G_{a}\right)^{\delta-d+1}$ (see $[21$, Theorem 11 and its corollary] or [22, Chapter V, Section 17]). The group $\mathcal{J}$ appears in the construction of the generalized Jacobian of a singular curve.

LEMMA 1. With the above notation, the following identities hold:

(1) $[\mathcal{J}]=(\mathbb{L}-1)^{d-1} \mathbb{L}^{\delta-d+1}$;

(2) $\left[\pi_{\underline{c}-\underline{1}}\left(\mathcal{O}^{\times}\right)\right]=(\mathbb{L}-1) \mathbb{L}^{\|\underline{c}\|-\delta-1}$;

(3) $\chi_{g}\left(\mathcal{O}^{\times}\right)=(\mathbb{L}-1) \mathbb{L}^{-\delta-1}$;

(4) $\chi_{g}(\mathcal{O})=\mathbb{L}^{-\delta}$.

Proof. (1) This identity follows from the fact that $\mathcal{J} \cong\left(k^{\times}\right)^{d-1} \times k^{\delta-d+1}$ as algebraic variety (see [22, Chapter V, Section 17]). 
(2) From the sequence of algebraic groups

$$
1 \rightarrow \mathcal{O}^{\times} /(\underline{1}+\mathcal{F}) \rightarrow \widetilde{\mathcal{O}}^{\times} /(\underline{1}+\mathcal{F}) \rightarrow \mathcal{J} \rightarrow 1
$$

we have $\left[\pi_{\underline{c}-1}\left(\mathcal{O}^{\times}\right)\right]=\left[\mathcal{O}^{\times} /(\underline{1}+\mathcal{F})\right]=[\mathcal{J}]^{-1}\left[\widetilde{\mathcal{O}}^{\times} /(\underline{1}+\mathcal{F})\right]$. Now, the result follows from $(1)$, since $\left[\widetilde{\mathcal{O}}^{\times} /(\underline{1}+\mathcal{F})\right]=(\mathbb{L}-1)^{d} \mathbb{L}^{\|\underline{c}\|-d}$.

(3) This identity follows from (2) by using $\chi_{g}\left(\mathcal{O}^{\times}\right)=\left[\pi_{\underline{c}-1}\left(\mathcal{O}^{\times}\right)\right] \mathbb{L}^{-\|\underline{c}\|}$.

(4) To prove this identity, we note that the exact sequence of (finite dimensional) vector spaces

$$
0 \rightarrow \mathcal{O} / \mathcal{F} \rightarrow \widetilde{\mathcal{O}} / \mathcal{F} \rightarrow \widetilde{\mathcal{O}} / \mathcal{O} \rightarrow 0
$$

implies that $[\mathcal{O} / \mathcal{F}]=[\widetilde{\mathcal{O}} / \mathcal{O}]^{-1}[\widetilde{\mathcal{O}} / \mathcal{F}]=\mathbb{L}^{\|\underline{c}\|-\delta}$. Therefore,

$$
\chi_{g}(\mathcal{O})=\left[\pi_{\underline{c}-\underline{1}}(\mathcal{O})\right] \mathbb{L}^{-\|\underline{c}\|}=[\mathcal{O} / \mathcal{F}] \mathbb{L}^{-\|\underline{c}\|}=\mathbb{L}^{-\delta} .
$$

\section{$\S 5$. Zeta functions for curve singularities}

In this section $k$ is a field of characteristic $p \geqslant 0$. For $\underline{n} \in S$ we set

$$
\mathcal{I}_{\underline{n}}:=\{I \subseteq \mathcal{O} \mid I=\underline{z} \mathcal{O}, \text { with } \underline{v}(\underline{z})=\underline{n}\},
$$

and for $m \in \mathbb{N}$,

$$
\mathcal{I}_{m}:=\bigcup_{\substack{\underline{n} \in S \\\|\underline{n}\|=m}} \mathcal{I}_{\underline{n}} .
$$

Lemma 2. For any $\underline{n} \in S$, there exists a bijection $\sigma_{\underline{n}}$ between $\mathcal{I}_{\underline{n}}$ and an algebraic subset $\sigma_{\underline{n}}\left(\mathcal{I}_{\underline{n}}\right)$ of $\mathcal{J}$, when $\mathcal{J}$ is considered as an algebraic variety. Furthermore, if $\underline{n} \geqslant \underline{c}$, then $\sigma_{\underline{n}}\left(\mathcal{I}_{\underline{n}}\right)=\mathcal{J}$.

Proof. Let $I=\underline{z} \mathcal{O}$ be a principal ideal $\mathcal{I}_{\underline{n}}$, with $\underline{z}=\underline{t} \underline{\underline{n}} \underline{\mu}, \underline{t} \underline{\underline{n}}=\left(t_{1}^{n_{1}}, \ldots, t_{d}^{n_{d}}\right)$ and $\underline{\mu}=\left(\mu_{1}, \ldots, \mu_{d}\right) \in \widetilde{\mathcal{O}}^{\times}$. Since $\underline{\mu}$ is determined up to an element of $\mathcal{O}^{\times}$, we may assume that $\underline{z}=\underline{t} \underline{\underline{n}} \underline{\mu} \underline{w}$, with $\underline{\mu} \in \mathcal{J}$ and $\underline{w} \in \mathcal{O}^{\times}$. Here we identify $\mathcal{J}$ with a fixed set of polynomial representatives, and thus $\underline{\mu}$ is one of these representatives. We define

$$
\begin{aligned}
\sigma_{\underline{n}}: \mathcal{I}_{\underline{n}} & \rightarrow \mathcal{J} \\
\underline{t} \underline{\underline{n}} \underline{\mu} \mathcal{O} & \rightarrow \underline{\mu} .
\end{aligned}
$$


Then $\sigma_{\underline{n}}$ is well defined as a one-to-one mapping. We now show that $\sigma_{\underline{n}}\left(\mathcal{I}_{\underline{n}}\right)$ is an algebraic subset of $\mathcal{J}$ whose points parametrize the ideals in $\mathcal{I}_{\underline{n}}$. Let $\underline{\mu}$ be a fixed element in $\mathcal{J}$; if $\underline{t}^{\underline{n}} \underline{\mu} \in \mathcal{O}$, then $\underline{t}^{\underline{n}} \underline{\mu}$ is the generator of an ideal in $\mathcal{I}_{\underline{n}}$. The condition " $\underline{\underline{n}} \underline{\underline{\mu}} \in \mathcal{\mathcal { O }}$ " is algebraic (see (2.1)); hence $\sigma_{\underline{n}}\left(\mathcal{I}_{\underline{n}}\right)$ is an algebraic subset of $\mathcal{J}$. Finally, if $\underline{n} \geqslant \underline{c}$, the condition $\underline{t}^{\underline{n}} \underline{\mu} \in \mathcal{O}$ is always true for any $\underline{\mu} \in \mathcal{J}$, and then $\sigma_{\underline{n}}\left(\mathcal{I}_{\underline{n}}\right)=\mathcal{J}$.

From now on we will identify $\mathcal{I}_{\underline{n}}$ with $\sigma_{\underline{n}}\left(\mathcal{I}_{\underline{n}}\right)$.

Since

$$
\mathcal{I}_{m}=\bigcup_{\{\underline{n} \in S \mid\|\underline{n}\|=m\}} \mathcal{I}_{\underline{n}},
$$

by applying Lemma 2 , we have that $\mathcal{I}_{m}$ is an algebraic subset of $\mathcal{J}$, for any $m \in \mathbb{N}$. By using this fact, the following two formal series are well defined.

Definition 5. We associate to $\mathcal{O}$ the two following zeta functions:

$$
Z\left(T_{1}, \ldots, T_{d}, \mathcal{O}\right):=\sum_{\underline{n} \in S}\left[\mathcal{I}_{\underline{n}}\right] \mathbb{L}^{-\|\underline{n}\|} T^{\underline{n}} \in \mathcal{M}_{k} \llbracket T_{1}, \ldots, T_{d} \rrbracket,
$$

where $T^{\underline{n}}:=T_{1}^{n_{1}} \times \cdots \times T_{d}^{n_{d}}$, and

$$
Z(T, \mathcal{O}):=Z(T, \ldots, T, \mathcal{O}) .
$$

Lemma 3. The sets $\{\underline{z} \in \mathcal{O} \mid \underline{v}(\underline{z})=\underline{n}\}, \underline{n} \in S$, and $\{\underline{z} \in \mathcal{O} \mid\|\underline{v}(\underline{z})\|=k\}$, $k \in \mathbb{N}$, are cylindric subsets of $\mathcal{O}$. In addition,

$$
\chi_{g}(\{\underline{z} \in \mathcal{O} \mid \underline{v}(\underline{z})=\underline{n}\})=\left[\mathcal{I}_{\underline{n}}\right]\left[\pi_{\underline{c}-\underline{1}}\left(\mathcal{O}^{\times}\right)\right] \mathbb{L}^{-\|\underline{n}+\underline{c}\|} .
$$

Proof. Every $\underline{x} \in \mathcal{O}$, with $\underline{v}(\underline{x})=\underline{n}$, can be expressed as

$$
\begin{aligned}
\underline{x} & =\underline{t}^{\underline{n}} \underline{\mu} \underline{w}, \quad \underline{\mu} \in \mathcal{J}, \underline{w} \in \mathcal{O}^{\times} \\
& =\underline{t}^{\underline{n}} \underline{\mu} \pi_{\underline{c}-1}(\underline{w})+\underline{t}^{\underline{n}+\underline{c}} \underline{y}, \quad \underline{y} \in \widetilde{\mathcal{O}} .
\end{aligned}
$$

Thus $x$ is determined by its $\underline{n}+\underline{c}$ jet, which in turn is determined by the condition

$$
\underline{\mu} \pi_{\underline{c}-\underline{1}}(\underline{w}) \in \mathcal{I}_{\underline{n}} \times \pi_{\underline{c}-\underline{1}}\left(\mathcal{O}^{\times}\right),
$$

which is a constructible one. Therefore, $\{\underline{z} \in \mathcal{O} \mid \underline{v}(\underline{z})=\underline{n}\}, \underline{n} \in S$, is a constructible set, and

$$
\chi_{g}(\{\underline{z} \in \mathcal{O} \mid \underline{v}(\underline{z})=\underline{n}\})=\left[\mathcal{I}_{\underline{n}} \times \pi_{\underline{c}-\underline{1}}\left(\mathcal{O}^{\times}\right)\right] \mathbb{L}^{-\|\underline{n}+\underline{c}\|} .
$$


Finally, $\{\underline{z} \in \mathcal{O} \mid\|\underline{v}(\underline{z})\|=k\}, k \in \mathbb{N}$, is cylindric, since

$$
\{\underline{z} \in \mathcal{O} \mid\|\underline{v}(\underline{z})\|=k\}=\bigcup_{\{\underline{n} \in S \mid\|\underline{n}\|=k\}}\{\underline{z} \in \mathcal{O} \mid \underline{v}(\underline{z})=\underline{n}\} .
$$

COROLlaRY 1. With the above notation, the following assertions hold.

(1) The functions

$$
\begin{aligned}
T^{\|\underline{v}(\cdot)\|}: & \mathcal{O} \rightarrow \mathbb{Z} \llbracket T \rrbracket \\
\underline{z} & \rightarrow T^{\|\underline{v}(\underline{z})\|},
\end{aligned}
$$

with $T^{\|\underline{v}(\underline{z})\|}:=0$, if $\|\underline{v}(\underline{z})\|=\infty$, and

$$
\begin{aligned}
T^{\underline{v}(\cdot)}: \mathcal{O} & \rightarrow \mathbb{Z} \llbracket T_{1}, \ldots, T_{d} \rrbracket \\
\underline{z} & \rightarrow T^{\underline{v}}(\underline{z})
\end{aligned}
$$

with $T^{\underline{v}}(\underline{z}):=0$, if $\|\underline{v}(\underline{z})\|=\infty$, are cylindric.

(2) $\left[\pi_{\underline{c}-\underline{1}}\left(\mathcal{O}^{\times}\right)\right] \mathbb{L}^{-\|\underline{c}\|} Z\left(T_{1}, \ldots, T_{d}, \mathcal{O}\right)=\int_{\mathcal{O}} T^{\underline{v}(\underline{z})} d \chi_{g}$.

(3) $\left[\pi_{\underline{c}-\underline{1}}\left(\mathcal{O}^{\times}\right)\right] \mathbb{L}^{-\|\underline{c}\|} Z(T, \mathcal{O})=\int_{\mathcal{O}} T^{\|\underline{v}(\underline{z})\|} d \chi_{g}$.

Proof. The assertions follow from Definition 3 by applying Lemma 3 .

Let $J_{\underline{n}}(\mathcal{O})=\{\underline{z} \in \mathcal{O} \mid \underline{v}(\underline{z}) \geqslant \underline{n}\}$, for $\underline{n} \in \mathbb{N}^{d}$, be an ideal. Since $J_{\underline{n}}(\mathcal{O}) \subseteq$ $J_{\underline{n}+1}(\mathcal{O})$, they give a multi-index filtration of the ring $\mathcal{O}$. Note that the $J_{\underline{n}}(\mathcal{O})$ are cylindric subsets of $\mathcal{O}$. As in [7], we introduce the following motivic Poincaré series.

Definition 6. The generalized Poincaré series of a multiindex filtration given by the ideals $J_{\underline{n}}(\mathcal{O})$ is the integral

$$
P_{g}\left(T_{1}, \ldots, T_{d}, \mathcal{O}\right):=\int_{\mathbb{P O}} T^{\underline{v}(\underline{z})} d \chi_{g} \in \mathcal{M}_{k} \llbracket T_{1}, \ldots, T_{d} \rrbracket .
$$

The generalized Poincaré series is related to the zeta function of Definition 5 as follows.

LEMMA 4. With the above notation,

$$
Z\left(T_{1}, \ldots, T_{d}, \mathcal{O}\right)=\mathbb{L}^{\delta+1} P_{g}\left(T_{1}, \ldots, T_{d}\right) .
$$

Proof. By Corollary 1(2), and Lemma 1(2),

$$
Z\left(T_{1}, \ldots, T_{d}, \mathcal{O}\right)=\frac{1}{(\mathbb{L}-1) \mathbb{L}^{-\delta-1}} \int_{\mathcal{O}} T^{\underline{v}(\underline{z})} d \chi_{g}=\mathbb{L}^{\delta+1} \int_{\mathbb{P O}} T^{\underline{v}(\underline{z})} d \chi_{g}
$$

(see Remark 4). 
We set $l(\underline{n}):=\operatorname{dim}_{k} \mathcal{O} / J_{\underline{n}}(\mathcal{O})$ and the vector $\underline{e}_{i} \in \mathbb{N}^{d}, i=1, \ldots, d$, to have all entries zero except for the $i$ th one, which is equal to one. Let $I_{0}:=\{1,2, \ldots, d\}$. For $I \subseteq I_{0}$, let $\# I$ be the number of elements of $I$. Let $\underline{1}_{I}$ be the element of $\mathbb{N}^{d}$ whose $i$ th component is equal to 1 or 0 if $i \in I$ or $i \notin I$, respectively. Note that $\underline{0}=\underline{1}_{\emptyset}$ and $\underline{1}=\underline{1}_{I_{0}}$.

REMARK 5. We recall that

$$
\underline{n} \in S \Longleftrightarrow \operatorname{dim}_{k} J_{\underline{n}}(\mathcal{O}) / J_{\underline{n}+\underline{e}_{i}}(\mathcal{O})=1, \quad \text { for any } i=1, \ldots, d
$$

(see, e.g., [10]). Thus, for $\underline{n} \in S$, and for any fixed $\underline{e}_{i_{0}}$, we have the following exact sequence of $k$-vector spaces:

$$
0 \rightarrow k \rightarrow J_{\underline{n}+\underline{e}_{i}}(\mathcal{O}) \rightarrow J_{\underline{n}}(\mathcal{O}) \rightarrow 0,
$$

where $J_{\underline{n}}(\mathcal{O}) / J_{\underline{n}}+\underline{e}_{i_{0}}(\mathcal{O}) \cong k$. Now, if $\underline{m} \geqslant \underline{n}+\underline{e}_{i_{0}}+\underline{1}$, from the previous exact sequence, one gets

$$
0 \rightarrow k \rightarrow J_{\underline{n}+\underline{e}_{i}}(\mathcal{O}) / t^{\underline{m}+1} \widetilde{\mathcal{O}} \rightarrow J_{\underline{n}}(\mathcal{O}) / t^{\underline{m}+\underline{1}} \widetilde{\mathcal{O}} \rightarrow 0,
$$

and hence

$$
\left[J_{\underline{n}}(\mathcal{O}) / t^{\underline{m}+1} \widetilde{\mathcal{O}}\right]=\mathbb{L}\left[J_{\underline{n}}+\underline{e}_{i_{0}}(\mathcal{O}) / t^{\underline{m}+1} \widetilde{\mathcal{O}}\right] .
$$

Proposition 1. We have $\left[\mathcal{I}_{\underline{n}}\right]=(\mathbb{L}-1)^{-1} \mathbb{L}\|\underline{n}\|+1 \sum_{I \subseteq I_{0}}(-1)^{\#(I)} \mathbb{L}^{-l\left(\underline{n}+\underline{1}_{I}\right)}$, for $\underline{n} \in S$.

Proof. We claim that

$$
\chi_{g}\left(J_{\underline{n}}(\mathcal{O})\right)= \begin{cases}\mathbb{L} \cdot \chi_{g}\left(J_{\underline{n}}+\underline{e}_{i_{0}}(\mathcal{O})\right), & \text { if } \operatorname{dim}_{k}\left(J_{\underline{n}}(\mathcal{O}) / J_{\underline{n}}+\underline{e}_{i_{0}}(\mathcal{O})\right)=1, \\ \chi_{g}\left(J_{\underline{n}}+\underline{e}_{i_{0}}(\mathcal{O})\right), & \text { if } \operatorname{dim}_{k}\left(J_{\underline{n}}(\mathcal{O}) / J_{\underline{n}}+\underline{e}_{i_{0}}(\mathcal{O})\right)=0\end{cases}
$$

for any $\underline{e}_{i_{0}}$. The formula is clear if $\operatorname{dim}_{k}\left(J_{\underline{\underline{n}}}(\mathcal{O}) / J_{\underline{\underline{n}}}+\underline{e}_{i_{0}}(\mathcal{O})\right)=0$, that is, if $J_{\underline{n}}(\mathcal{O})=J_{\underline{n}+\underline{e}_{i}}(\mathcal{O})$; thus we can assume that $\operatorname{dim}_{k}\left(J_{\underline{n}}(\mathcal{O}) / J_{\underline{n}}+\underline{e}_{i_{0}}(\mathcal{O})\right)=1$, that is, that $\underline{n} \in S$. By taking $\underline{m}$ as in Remark 5 , one gets

$$
\begin{aligned}
\chi_{g}\left(J_{\underline{n}}(\mathcal{O})\right) & =\mathbb{L}^{-\|\underline{m}+\underline{1}\|}\left[J_{\underline{\underline{n}}}(\mathcal{O}) / t^{\underline{m}+\underline{1} \widetilde{\mathcal{O}}]}=\mathbb{L}\left(\mathbb{L}^{-\|\underline{m}+\underline{1}\|}\left[J_{\underline{\underline{n}}+\underline{e}_{i_{0}}}(\mathcal{O}) / t^{\underline{m}+\underline{1}} \widetilde{\mathcal{O}}\right]\right)\right. \\
& =\mathbb{L} \cdot \chi_{g}\left(J_{\underline{\underline{n}}+\underline{e}_{i_{0}}}(\mathcal{O})\right) .
\end{aligned}
$$

Now we fix a sequence of the form

$$
\underline{0}=\underline{m}_{0} \leqslant \underline{m}_{1} \leqslant \cdots \leqslant \underline{m}_{j} \leqslant \underline{m}_{j+1} \leqslant \cdots \leqslant \underline{m}_{k}=\underline{n},
$$


where $\underline{m}_{j+1}=\underline{m}_{j}+\underline{e}_{j}$, for $j=0, \ldots, k-1$. Then by applying (5.3) we have

$$
\chi_{g}\left(J_{\underline{n}}(\mathcal{O})\right)=\mathbb{L}^{-l(\underline{n})} \cdot \chi_{g}(\mathcal{O}) .
$$

On the other hand,

$$
\begin{aligned}
\chi_{g}(\{\underline{z} \in \mathcal{O} \mid \underline{v}(\underline{z})=\underline{n}\}) & =\chi_{g}\left(J_{\underline{n}}(\mathcal{O}) \backslash \bigcup_{i=1}^{d} J_{\underline{n}+\underline{e}_{i}}(\mathcal{O})\right) \\
& =\chi_{g}\left(J_{\underline{n}}(\mathcal{O})\right)-\chi_{g}\left(\bigcup_{i=1}^{d} J_{\underline{n}+\underline{e}_{i}}(\mathcal{O})\right) .
\end{aligned}
$$

Now by using the identities

$$
\begin{aligned}
& \chi_{g}\left(\bigcup_{i=1}^{n} A_{i}\right)=\sum_{\substack{J \subseteq\{1,2, \ldots, n\} \\
J \neq \emptyset}}(-1)^{\#(J)-1} \chi_{g}\left(\bigcap_{j \in J} A_{j}\right), \\
& J_{\underline{n}+\underline{e}_{i_{1}}}(\mathcal{O}) \cap \cdots \cap J_{\underline{n}+\underline{e}_{i j}}(\mathcal{O})=J_{\underline{n}+\underline{e}_{i_{1}}+\cdots+\underline{e}_{i_{j}}}(\mathcal{O}),
\end{aligned}
$$

(5.4), and Lemma 3, we obtain

$$
\begin{aligned}
{\left[\mathcal{I}_{\underline{n}}\right] } & =\frac{\mathbb{L}^{\|\underline{n}+\underline{c}\|}}{\left[\pi_{\underline{c}-\underline{1}}\left(\mathcal{O}^{\times}\right)\right]}\left(\chi_{g}\left(J_{\underline{n}}(\mathcal{O})\right)-\sum_{\substack{I \subseteq\{1,2, \ldots, d\} \\
I \neq \emptyset}}(-1)^{\#(I)-1} \chi_{g}\left(J_{\underline{n}+\sum_{i \in I} \underline{e}_{i}}(\mathcal{O})\right)\right) \\
& =\frac{\mathbb{L}^{\|\underline{n}+\underline{c}\|} \chi_{g}(\mathcal{O})}{\left[\pi_{\underline{c}-1}\left(\mathcal{O}^{\times}\right)\right]}\left(\mathbb{L}^{-l(\underline{n})}-\sum_{\substack{I \subseteq\{1,2, \ldots, d\} \\
I \neq \emptyset}}(-1)^{\#(I)-1} \mathbb{L}^{-l\left(\underline{n}+\underline{1}_{I}\right)}\right) .
\end{aligned}
$$

Finally, the result follows from the previous identity by using

$$
\left[\pi_{\underline{c}-\underline{1}}\left(\mathcal{O}^{\times}\right)\right]=(\mathbb{L}-1) \mathbb{L}^{\|\underline{c}\|-\delta-1}, \quad \chi_{g}(\mathcal{O})=\mathbb{L}^{-\delta}
$$

(see Lemma 1).

REMARK 6 . Let $k$ be a field of characteristic $p>0$. Let $Y$ be an algebraic curve defined over $k$. Let $\mathcal{O}_{P, Y}$ be the local ring of $Y$ at the point $P$, and let $\widehat{O}_{P, Y}$ be its completion. Then $\mathcal{J} \cong\left(G_{m}\right)^{d-1} \times \Gamma$, where $\Gamma$ is a subgroup of a product of groups of Witt vectors of finite length. If $p \geqslant c_{i}$, for $i=1, \ldots, d$, where $\underline{c}=\left(c_{1}, \ldots, c_{d}\right)$ is the conductor of the semigroup of $\widehat{O}_{P, Y}$, then $\mathcal{J} \cong\left(G_{m}\right)^{\bar{d}-1} \times\left(G_{a}\right)^{\delta-d+1}$ (see [22, Proposition 9, Chapter V, Section 16]). We can attach to $\widehat{O}_{P, Y}$ a zeta function $Z\left(T_{1}, \ldots, T_{d}, \widehat{O}_{P, Y}\right)$ defined as before. All the results presented so far are valid in this context, in particular Proposition 1. 
§6. Rationality of $Z\left(T_{1}, \ldots, T_{d}, \mathcal{O}\right)$

From now on $k$ is a field of characteristic $p \geqslant 0$, and $\mathcal{O}$ is a totally rational ring as before. The aim of this section is to prove the rationality of the zeta function $Z\left(T_{1}, \ldots, T_{d}, \mathcal{O}\right)$ and, subsequently, of the generalized Poincaré series $P_{g}\left(T_{1}, \ldots, T_{d}, \mathcal{O}\right)$ by Lemma 4 , giving also an explicit formula for it.

We start by establishing the notation and preliminary results required in the proof.

Set $I_{0}:=\{1,2, \ldots, d\}$, and for a subset $J$ of $I_{0}$, set

$$
H_{J}:=\left\{\underline{n} \in S \mid n_{j} \geqslant c_{j} \Leftrightarrow j \in J\right\},
$$

where $\underline{c}=\left(c_{1}, \ldots, c_{d}\right)$ is the conductor of $S$, and also

$$
H_{J}(\mathcal{O}):=\left\{\underline{z} \in \mathcal{O} \mid \underline{v}(\underline{z}) \in H_{J}\right\}
$$

Note that $H_{\emptyset}(\mathcal{O})=\left\{\underline{z} \in \mathcal{O} \mid 0 \leqslant v\left(z_{i}\right) \leqslant c_{i}-1, i=1, \ldots, d\right\}$ and that $H_{I_{0}}(\mathcal{O})=\mathcal{F}$. Given $\underline{m} \in \mathbb{N}^{d}$ such that $\underline{c}>\underline{m}$, that is, $c_{i}>m_{i}$, for $i=1, \ldots, d$, set

$$
\begin{gathered}
H_{J, \underline{m}}:=\left\{\underline{n} \in S \mid n_{j} \geqslant c_{j} \text { if } j \in J, \text { and } n_{j}=m_{j} \text { if } j \notin J\right\}, \\
H_{J, \underline{m}}(\mathcal{O}):=\left\{\underline{z} \in \mathcal{O} \mid \underline{v}(\underline{z}) \in H_{J, \underline{m}}\right\},
\end{gathered}
$$

and for a fixed $J$ satisfying $\emptyset \subsetneq J \subsetneq I_{0}$, set

$$
B_{J}:=\left\{\underline{m} \in \mathbb{N}^{\# J} \mid H_{J, \underline{m}} \neq \emptyset\right\} .
$$

Therefore, for $\emptyset \subsetneq J \subsetneq I_{0}$, one gets the following partition for $H_{J}(\mathcal{O})$ :

$$
H_{J}(\mathcal{O})=\bigcup_{\underline{m} \in B_{J}} H_{J, \underline{m}}(\mathcal{O}) .
$$

LEMMA 5. With the above notation, the following assertions hold.

(1) Let $J=\{1, \ldots, r\}$ with $1 \leqslant r<d$, and let $\underline{m} \in \mathbb{N}^{d}$ such that $\underline{c}>\underline{m}$. If $H_{J, \underline{m}} \neq \emptyset$, then

$$
H_{J, \underline{m}}=\left\{\begin{array}{l|l}
\underline{n} \in \mathbb{N}^{d} & \begin{array}{l}
n_{i} \geqslant c_{i}, \text { for } i=1, \ldots, r \\
n_{i}=m_{i}, \text { for } i=r+1, \ldots, d
\end{array}
\end{array}\right\} .
$$


(2) $H_{J, \underline{m}}(\mathcal{O})$ and $H_{J}(\mathcal{O})$ are cylindric subsets of $\mathcal{O}$.

Proof. (1) Since $H_{J, \underline{m}} \neq \emptyset$, there exist $\underline{f}(\underline{m}):=\left(e_{1}, \ldots, e_{r}, m_{r+1}, \ldots, m_{d}\right) \in$ $H_{J, \underline{m}}$ and $\underline{z}=\left(z_{1}, \ldots, z_{d}\right) \in \mathcal{O}$ such that

$$
z_{i}= \begin{cases}\sum_{k=e_{i}}^{\infty} a_{k, i} t_{i}^{k}, & \text { with } a_{e_{i}, i} \neq 0, \text { for } i=1, \ldots, r \\ \sum_{k=m_{i}}^{\infty} a_{k, i} t_{i}^{k}, & \text { with } a_{m_{i}, i} \neq 0, \text { for } i=r+1, \ldots, d\end{cases}
$$

Since $\mathcal{O}$ is a cylindric subset of $\widetilde{\mathcal{O}}$ defined by the condition $\Delta=0($ see $(2.1))$ that involves only the variables $a_{k, i}$ with $0 \leqslant k<c_{k}, k=1, \ldots, d$, it follows that any $\underline{y}=\left(y_{1}, \ldots, y_{d}\right) \in \widetilde{\mathcal{O}}$ of the form

$$
y_{i}= \begin{cases}\sum_{k=c_{i}}^{\infty} a_{k, i} t_{i}^{k}, & \text { for } i=1, \ldots, r \\ \sum_{k=m_{i}}^{\infty} a_{k, i} t_{i}^{k}, & \text { with } a_{m_{i}, i} \neq 0, \text { for } i=r+1, \ldots, d,\end{cases}
$$

belongs to $\mathcal{O}$, and therefore

$$
H_{J, \underline{m}}=\left\{\begin{array}{l|l}
\underline{n} \in \mathbb{N}^{d} & \begin{array}{l}
n_{i} \geqslant c_{i}, \text { for } i=1, \ldots, r \\
n_{i}=m_{i}, \text { for } i=r+1, \ldots, d
\end{array}
\end{array}\right\} .
$$

(2) Since $H_{J}(\mathcal{O})$ is a finite disjoint union of subsets of the form $H_{J, \underline{m}}(\mathcal{O})$, it is sufficient to show that $H_{J, \underline{m}}(\mathcal{O})$ is a cylindric subset of $\mathcal{O}$. On the other hand, since $H_{J, \underline{m}}(\mathcal{O})=\mathcal{O} \cap\left\{\underline{z} \in \widetilde{\mathcal{O}} \mid \underline{v}(\underline{z}) \in H_{J, \underline{m}}\right\}$ and $\mathcal{O}$ is a cylindric subset of $\widetilde{\mathcal{O}}$, it is enough to show that $\underline{v}(\underline{z}) \in H_{J, \underline{m}}$ is a constructible condition in $J \frac{l}{\widetilde{\mathcal{O}}}$, for some $\underline{l} \in \mathbb{N}^{d}$. Let $\underline{l}=\left(c_{1}, \ldots, c_{r}, m_{r+1}+1, \ldots, m_{d}+1\right)$, and let $\underline{z}=\left(z_{1}, \ldots, z_{d}\right) \in \widetilde{\mathcal{O}}$ with $z_{i}=\sum_{k=0}^{\infty} a_{k, i} t_{i}^{k}$, for $i=1, \ldots, d$. Since

$$
v_{i}\left(z_{i}\right)=m_{i} \Leftrightarrow\left\{\begin{array}{l}
a_{k, i}=0, \quad k=0, \ldots, m_{i}-1, \\
a_{m_{i}, i} \neq 0
\end{array}\right.
$$

and

$$
v_{i}\left(z_{i}\right) \geqslant c_{i} \Leftrightarrow\left\{a_{k, i}=0, \quad k=0, \ldots, c_{i}-1\right.
$$

$\underline{v}(\underline{z}) \in H_{J, \underline{m}}$ is a constructible condition in $J \frac{l}{\widetilde{\mathcal{O}}}$.

REMARK 7. Let $J=\{1, \ldots, r\}$ with $1 \leqslant r<d$, and let $\underline{m} \in \mathbb{N}^{d}$ such that $\underline{c}>\underline{m}$. If $H_{J, \underline{m}} \neq \emptyset$, then $\left[\mathcal{I}_{\underline{k}}\right]=\left[\mathcal{I}_{\underline{f_{J}}(\underline{m})}\right]$, with $\underline{f_{J}}(\underline{m})=\left(c_{1}, \ldots, c_{r}\right.$, $\left.m_{r+1}, \ldots, m_{d}\right)$, for any $\underline{k} \in H_{J, \underline{m}}$. 
The remark follows from the following observation. With the notation used in the proof of Lemma 2, the following conditions are equivalent:

$$
\begin{aligned}
\sigma_{\underline{k}}(I)=\underline{\mu}, \underline{k} \in H_{J, \underline{m}} & \Leftrightarrow t^{\underline{k}} \underline{\mu} \underline{v} \in \mathcal{O}, \quad \text { for any } \underline{v} \in \mathcal{O}^{\times}, \underline{k} \in H_{J, \underline{m}} \\
& \Leftrightarrow t \underline{f_{J}} \underline{\underline{m})} \underline{\mu} \underline{v} \in \mathcal{O}, \quad \text { for any } \underline{v} \in \mathcal{O}^{\times} .
\end{aligned}
$$

In the proof of the last equivalence, we use the same reasoning as that used in the proof of Lemma 5(1).

LEMMA 6. Let $J$ be a nonempty and proper subset of $I_{0}$, such that $H_{J, \underline{m}}(\mathcal{O}) \neq \emptyset$. Then

$$
\int_{H_{J, \underline{m}}(\mathcal{O})} T^{\underline{v}(\underline{z})} d \chi_{g}=\frac{\left[\mathcal{I}_{f_{J}(\underline{m})}\right]\left[\pi_{\underline{c}-\underline{1}}\left(\mathcal{O}^{\times}\right)\right] \mathbb{L}^{-\|\underline{c}\|-\left\|\underline{f_{J}}(\underline{m})\right\|_{T}} T_{\underline{f_{J}}}(\underline{m})}{\prod_{i=1}^{r}\left(1-\mathbb{L}^{-1} T_{i}\right)},
$$

where $\underline{f_{J}}(\underline{m})=\left(c_{1}, \ldots, c_{r}, m_{r+1}, \ldots, m_{d}\right) \in S$, with $m_{i}<c_{i}, r+1 \leqslant i \leqslant d$.

Proof. Without loss of generality, we assume that $J=\{1, \ldots, r\}$, with $1 \leqslant r<d$. With this notation, by using $H_{J, \underline{m}}(\mathcal{O}) \neq \emptyset$ and Lemma $5(1)$, we have

$$
H_{J, \underline{m}}=\left\{\begin{array}{l|l}
\underline{n} \in \mathbb{N}^{d} & \begin{array}{l}
n_{i} \geqslant c_{i}, \text { for } i=1, \ldots, r, \\
n_{i}=m_{i}, \text { for } i=r+1, \ldots, d
\end{array}
\end{array}\right\} .
$$

Now, by using Lemma 3 and Remark 7, we have

$$
\begin{aligned}
& \int_{H_{J, \underline{m}}(\mathcal{O})} T^{\underline{v}(\underline{z})} d \chi_{g}=\left[\mathcal{I}_{f_{J}(\underline{m})}\right]\left[\pi_{\underline{c}-\underline{1}}\left(\mathcal{O}^{\times}\right)\right] \mathbb{L}^{-\|\underline{c}\|-\| \underline{f_{J}}} \underline{\underline{m}) \|} T^{f_{J}}(\underline{m})\left(\sum_{e \in \mathbb{N}^{r}} \mathbb{L}^{-\|\underline{e}\|} T^{\underline{e}}\right) \\
& =\frac{\left[\mathcal{I}_{\underline{f}_{J}(\underline{m})}\right]\left[\pi_{\underline{c}-1}\left(\mathcal{O}^{\times}\right)\right] \mathbb{L}^{-\|\underline{c}\|-\left\|\underline{f_{J}}(\underline{m})\right\|_{T} \underline{f_{J}}(\underline{m})}}{\prod_{i=1}^{r}\left(1-\mathbb{L}^{-1} T_{i}\right)}
\end{aligned}
$$

where $\underline{f_{J}}(\underline{m})=\left(c_{1}, \ldots, c_{r}, m_{r+1}, \ldots, m_{d}\right) \in S$, with $m_{i}<c_{i}, r+1 \leqslant i \leqslant d$.

THEOREM 1. Let $k$ be a field of characteristic $p \geqslant 0$, and let $\mathcal{O}$ be a totally rational ring as before. Then (1)

$$
\begin{aligned}
& Z\left(T_{1}, \ldots, T_{d}, \mathcal{O}\right) \\
& =\sum_{\substack{n \in S \\
\underline{0} \leqslant \underline{n}<\underline{c}}}\left[\mathcal{I}_{\underline{n}}\right] \mathbb{L}^{-\|\underline{n}\|} T^{\underline{n}} \\
& +\sum_{\emptyset \subsetneq J \subsetneq I_{0}} \sum_{\underline{m} \in B_{J}}\left[\mathcal{I}_{\underline{f}_{J}(\underline{m})}\right]\left[\pi_{\underline{c}-\underline{1}}\left(\mathcal{O}^{\times}\right)\right] \mathbb{L}^{-\|\underline{c}\|-\left\|\underline{f_{J}}(\underline{m})\right\|} \frac{T \underline{f_{J}(\underline{m})}}{\prod_{i=1}^{r_{J}}\left(1-\mathbb{L}^{-1} T_{i}\right)}
\end{aligned}
$$




$$
+[\mathcal{J}] \mathbb{L}^{-\|\underline{c}\|} \frac{T^{\underline{c}}}{\prod_{i=1}^{d}\left(1-\mathbb{L}^{-1} T_{i}\right)},
$$

where $\underline{f_{J}}(\underline{m})=\left(c_{1}, \ldots, c_{r_{J}}, m_{r_{J}+1}, \ldots, m_{d}\right) \in S$, with $m_{i}<c_{i}, r_{J}+1 \leqslant i \leqslant d$, and $1 \leqslant r_{J}<d$.

$(2)$

$$
Z\left(T_{1}, \ldots, T_{d}, \mathcal{O}\right)=\frac{M\left(T_{1}, \ldots, T_{d}, \mathcal{O}\right)}{\prod_{i=1}^{d}\left(1-\mathbb{L}^{-1} T_{i}\right)},
$$

where $M\left(T_{1}, \ldots, T_{d}, \mathcal{O}\right)$ is a polynomial in $\mathcal{M}_{k}\left[T_{1}, \ldots, T_{d}\right]$ of degree at most $\|\underline{c}\|$ that satisfies $M(\mathbb{L}, \ldots, \mathbb{L}, \mathcal{O})=[\mathcal{J}]$.

Proof. Since $Z\left(T_{1}, \ldots, T_{d}, \mathcal{O}\right)=\left[\pi_{\underline{c}-1}\left(\mathcal{O}^{\times}\right)\right]^{-1} \mathbb{L}^{\| \underline{c}} \|_{\mathcal{O}_{\mathcal{O}}} T^{\underline{v}(\underline{z})} d \chi_{g}$ (see Corollary $1(2))$ and $\mathcal{O}=\bigcup_{J \subseteq I_{0}} H_{J}(\mathcal{O})$ is a disjoint union of cylindric subsets (see Lemma $5(2)), Z\left(T_{1}, \ldots, T_{d}, \mathcal{O}\right)$ is equal to a finite sum of integrals of type

$$
Z_{H_{J}}\left(T_{1}, \ldots, T_{d}, \mathcal{O}\right):=\left[\pi_{\underline{c}-\underline{1}}\left(\mathcal{O}^{\times}\right)\right]^{-1} \mathbb{L}^{\|\underline{c}\|} \int_{H_{J}(\mathcal{O})} T^{\underline{v}(\underline{z})} d \chi_{g} .
$$

In the case in which $J=\emptyset$,

$$
Z_{H_{\emptyset}}\left(T_{1}, \ldots, T_{d}, \mathcal{O}\right)=\sum_{\substack{\underline{n} \in S \\ \underline{0} \leqslant \underline{n}<\underline{c}}}\left[\mathcal{I}_{\underline{n}}\right] \mathbb{L}^{-\|\underline{n}\|} T^{\underline{n}} \in \mathcal{M}_{k}\left[T_{1}, \ldots, T_{d}\right],
$$

and the degree of $Z_{H_{\emptyset}}\left(T_{1}, \ldots, T_{d}, \mathcal{O}\right)$ is less than or equal to $\|\underline{c}\|-d$.

In the case $J=I_{0}$, by using Lemma 2 , we have

$$
Z_{H_{I_{0}}}\left(T_{1}, \ldots, T_{d}, \mathcal{O}\right)=[\mathcal{J}] \mathbb{L}^{-\|\underline{c}\|} \frac{T^{\underline{c}}}{\prod_{i=1}^{d}\left(1-\mathbb{L}^{-1} T_{i}\right)} .
$$

In the case in which $\emptyset \subsetneq J \subsetneq I_{0}$, we use the fact that $H_{J}(\mathcal{O})$ is a finite disjoint union of cylindric sets of the form $H_{J, \underline{m}}(\mathcal{O})$ (see $(6.1)$ ) to reduce the problem to the computation of the following integral:

$$
\begin{aligned}
Z_{H_{J, \underline{m}}}\left(T_{1}, \ldots, T_{d}, \mathcal{O}\right) & :=\left[\pi_{\underline{c}-\underline{1}}\left(\mathcal{O}^{\times}\right)\right]^{-1} \mathbb{L}^{\|\underline{c}\|} \int_{H_{J, \underline{m}}(\mathcal{O})} T^{\underline{v}(\underline{z})} d \chi_{g} \\
& =\frac{\left[\mathcal{I}_{\underline{f J}_{J}(\underline{m})}\right] \mathbb{L}^{-\left\|\underline{f_{J}}(\underline{m})\right\|_{T}} T_{\underline{f_{J}}(\underline{m})}}{\prod_{i=1}^{r_{J}}\left(1-\mathbb{L}^{-1} T_{i}\right)}
\end{aligned}
$$

(see Lemma 6), where $\underline{f_{J}}(\underline{m})=\left(c_{1}, \ldots, c_{r_{J}}, m_{r_{J}+1}, \ldots, m_{d}\right) \in S$, with $m_{i}<$ $c_{i}, r_{J}+1 \leqslant i \leqslant d$, and $1 \leqslant r_{J}<d$. Now the announced explicit formula follows from (6.2)-(6.5) above and the second part of the theorem is a straight consequence of it. 
Corollary 2. The zeta function $Z(T, \mathcal{O})$ is a rational function of the form

$$
Z(T, \mathcal{O})=\frac{R(T, \mathcal{O})}{\left(1-\mathbb{L}^{-1} T\right)^{d}},
$$

where $R(T, \mathcal{O})$ is a polynomial in $\mathcal{M}_{k}[T]$ of degree at most $\|\underline{c}\|$ that satisfies $R(\mathbb{L}, \mathcal{O})=[\mathcal{J}]$

Corollary 3. The generalized Poincaré series is a rational function of the form

$$
P_{g}\left(T_{1}, \ldots, T_{d}, \mathcal{O}\right)=\frac{Q\left(T_{1}, \ldots, T_{d}, \mathcal{O}\right)}{\prod_{i=1}^{d}\left(1-\mathbb{L}^{-1} T_{i}\right)},
$$

where $Q\left(T_{1}, \ldots, T_{d}, \mathcal{O}\right)$ is a polynomial in $\mathcal{M}_{k}\left[T_{1}, \ldots, T_{d}\right]$ of degree at most $\|\underline{c}\|$ that satisfies $Q(\mathbb{L}, \ldots, \mathbb{L}, \mathcal{O})=\mathbb{L}^{-\delta-1}[\mathcal{J}]$.

Definition 7. Let $k$ be a field of characteristic $p \geqslant 0$. Let $\mathcal{O}=\widehat{O}_{P, Y}$, where $Y$ is an algebraic curve over $k$ and $P$ is a singular point of $Y$. We say that $k$ is big enough for $Y$ if, for every singular point $P$ in $Y$, the following two conditions hold: (1) $\mathcal{O}$ is totally rational, and $(2) \mathcal{J} \cong$ $\left(G_{m}\right)^{d-1} \times\left(G_{a}\right)^{\delta-d+1}$.

Note that by Remark 6 , the condition " $k$ is big enough for $Y$ " is fulfilled when $p$ is big enough.

Corollary 4. Let $k$ be a field of characteristic $p \geqslant 0$. Let $\mathcal{O}=\widehat{O}_{P, Y}$, where $Y$ is an algebraic curve over $k$ and $P$ is a singular point of $Y$. If $k$ is big enough for $Y$, then $Z\left(T_{1}, \ldots, T_{d}, \mathcal{O}\right)$ is completely determined by the semigroup of $\mathcal{O}$.

Proof. By the explicit formula of Theorem $1, Z\left(T_{1}, \ldots, T_{d}, \mathcal{O}\right)$ is a rational function in the variables $T_{1}, \ldots, T_{d}$ and $\mathbb{L}$, depending on $S,\left[\pi_{\underline{c}-1}\left(\mathcal{O}^{\times}\right)\right]$, $[\mathcal{J}]$, and $\left[\mathcal{I}_{\underline{m}}\right]$ for $\|\underline{m}\|<\|\underline{c}\|$. In characteristic zero, $S$ determines uniquely $\left[\pi_{\underline{c}-\underline{1}}\left(\mathcal{O}^{\times}\right)\right],[\mathcal{J}],\left[\mathcal{I}_{\underline{m}}\right]$ for $\|\underline{m}\|<\|\underline{c}\|$ (see Lemma 1 and Proposition 1 ). If the characteristic is $p>0$, the hypothesis " $k$ is big enough for $Y$ " is required to assure that $[\mathcal{J}]$ is determined by the semigroup of $\mathcal{O}$.

\section{§7. Additive invariants and specialization of zeta functions}

Definition 8. Set $k=\mathbb{C}$. Consider a semigroup $S \subset \mathbb{N}^{d}$ such that $S=$ $S(\mathcal{O})$ for some $\mathcal{O}=\widehat{O}_{X, P}$, where $X$ is an algebraic curve over $\mathbb{C}$ and $P$ is a 
singular point of $X$. We set

$$
\mathcal{I}_{\underline{n}}(U):=(U-1)^{-1} U^{\|\underline{n}\|+1} \sum_{I \subseteq I_{0}}(-1)^{\#(I)} U^{-l\left(\underline{n}+\underline{1}_{I}\right)}, \quad \text { for } \underline{n} \in S,
$$

and

$$
\begin{aligned}
\mathcal{Z}\left(T_{1}, \ldots, T_{d}, U, S\right) & \\
:= & \sum_{\underline{n} \in S} \mathcal{I}_{\underline{n}}(U) U^{-\|\underline{n}\|} T^{\underline{n}} \\
& \quad \underline{\underline{n}<\underline{c}}{ } \\
& +\sum_{\emptyset \subsetneq J \subsetneq I_{0} \underline{m} \in B_{J}} \sum(U-1) U^{\|\underline{c}\|-\delta-1} \mathcal{I}_{f_{J}(\underline{m})}(U) U^{-\|\underline{c}\|-\left\|\underline{f_{J}}(\underline{m})\right\|} \\
& \times \frac{T \underline{f_{J}}(\underline{m})}{\prod_{i=1}^{r_{J}}\left(1-U^{-1} T_{i}\right)} \\
& +(U-1)^{d-1} U^{\delta-d+1} U^{-\|\underline{c}\|} \frac{T \underline{c}}{\prod_{i=1}^{d}\left(1-U^{-1} T_{i}\right)},
\end{aligned}
$$

where $f_{J}(\underline{m})=\left(c_{1}, \ldots, c_{r_{J}}, m_{r_{J}+1}, \ldots, m_{d}\right) \in S$, with $m_{i}<c_{i}, r_{J}+1 \leqslant i \leqslant d$, and $1 \leqslant r_{J}<d$, are as in the explicit formula given in Theorem 1(1); and $U$ is an indeterminate. We call $\mathcal{Z}\left(T_{1}, \ldots, T_{d}, U, S\right)$ the universal zeta function associated to $S$.

By definition, $\mathcal{Z}\left(T_{1}, \ldots, T_{d}, U, S\right)$ is completely determined by $S$.

LEMma 7. Assume that $k$ is big enough for $Y$. If $S=S(\mathcal{O})$, then

$$
Z\left(T_{1}, \ldots, T_{d}, \mathcal{O}\right)=\left.\mathcal{Z}\left(T_{1}, \ldots, T_{d}, U, S\right)\right|_{U=\left[\mathbb{A}_{k}^{1}\right]} .
$$

Proof. The result follows from Corollary 4.

REMARK 8. Let $R$ be a ring. An additive invariant is a map $\lambda: \operatorname{Var}_{k} \rightarrow R$ that satisfies the same conditions given in the definition of the Grothendieck symbol in the category of $k$-algebraic varieties (see, e.g., [18], [25]). By construction, the map $\operatorname{Var}_{k} \rightarrow K_{0}\left(\operatorname{Var}_{k}\right): V \mapsto[V]$ is a universal additive invariant; that is, the composition with [.] gives a bijection between the ring morphisms $K_{0}\left(\operatorname{Var}_{k}\right) \rightarrow R$ and additive invariants $\operatorname{Var}_{k} \rightarrow R$.

In the complex case, the Euler characteristic

$$
\chi(X)=\sum_{i}(-1)^{i} \operatorname{rank}\left(H_{c}^{i}(X(\mathbb{C}), \mathbb{C})\right)
$$


gives rise to an additive invariant $\chi: \operatorname{Var}_{\mathbb{C}} \rightarrow \mathbb{Z}$. Since $\chi\left(\mathbb{A}_{\mathbb{C}}^{1}\right)=1$, the Euler characteristic extends to a morphism $\mathcal{M}_{\mathbb{C}} \rightarrow \mathbb{Z}$. Then by specializing [.] to $\chi(\cdot)$ in (5.1) and (5.2), we obtain two "topological zeta functions," denoted by $\chi\left(Z\left(T_{1}, \ldots, T_{d}, \mathcal{O}\right)\right)$ and $\chi(Z(T, \mathcal{O}))$. From a computational point of view, these specializations are obtained by replacing $\mathbb{L}$ by $\mathbf{1}$ in the corresponding expressions.

REMARK 9. Let $(X, 0) \subset\left(\mathbb{C}^{2}, 0\right)$ be a reduced plane curve singularity defined by an equation $f=0$, with $f \in \mathcal{O}_{\left(\mathbb{C}^{2}, 0\right)}$ reduced. Let $h_{f}: V_{f} \rightarrow V_{f}$ be the monodromy transformation of the singularity $f$ acting on its Milnor fiber $V_{f}$ (see [1]). The zeta function of $h_{f}$ (also called zeta function of the monodromy) is defined to be

$$
\varsigma_{f}(T):=\prod_{i \geqslant 0}\left[\operatorname{det}\left(\mathrm{id}-\left.T \cdot\left(h_{f}\right)_{*}\right|_{H_{i}\left(V_{f} ; \mathbb{C}\right)}\right)\right]^{(-1)^{i+1}} .
$$

The following theorem is due to Campillo, Delgado, and Gusein-Zade ([4, Theorem 1]).

Theorem 2. ([4]) Set $k=\mathbb{C}$. Then for any $\mathcal{O}=\mathcal{O}_{\left(\mathbb{C}^{2}, 0\right)} /(f)$, with $f \in$ $\mathcal{O}_{\left(\mathbb{C}^{2}, 0\right)}$ reduced, and for any $S=S(\mathcal{O})$, we have

$$
\varsigma_{f}(T)=\mathcal{Z}\left(T_{1}, \ldots, T_{d}, U, S\right) \underset{U=1}{\left.\right|_{1}=\cdots=T_{d}=T} .
$$

Proof. As a consequence of the results of Campillo, Delgado, and GuseinZade (see [5], [6], [7]) and Lemma 4, we have $\chi(Z(T, \mathcal{O}))=\varsigma_{f}(T)$, the zeta function of the monodromy $\varsigma_{f}(T)$ associated to the germ of function $f:\left(\mathbb{C}^{2}, 0\right) \rightarrow(\mathbb{C}, 0)$. By Remark 9 and Lemma 7 , we have

$$
\chi(Z(T, \mathcal{O}))=\left.Z(T, \mathcal{O})\right|_{\mathbb{L} \rightarrow 1}=\left.\mathcal{Z}\left(T_{1}, \ldots, T_{d}, U, S\right)\right|_{\substack{T_{1}=\ldots=T_{d}=T \\ U=1}} .
$$

REMARK 10. In [29] the second author introduced a Dirichlet series $Z(\mathrm{Ca}(Y), T)$ associated to the effective Cartier divisors on an algebraic curve defined over a finite field $k=\mathbb{F}_{q}$. This zeta function admits an Euler product of the form

$$
Z(\mathrm{Ca}(Y), T)=\prod_{P \in X} Z_{\mathrm{Ca}(Y)}\left(T, q, O_{P, Y}\right),
$$


with

$$
Z_{\mathrm{Ca}(Y)}\left(T, q, O_{P, Y}\right):=Z_{\mathrm{Ca}(Y)}\left(T, O_{P, Y}\right)=\sum_{I \subseteq O_{P, Y}} T^{\operatorname{dim}_{k}\left(O_{P, Y} / I\right)},
$$

where $I$ runs through all the nonzero principal ideals of $O_{P, Y}$. The notation used here for the local factors of $Z(\mathrm{Ca}(Y), T)$ is slightly different from that used in [29]. In addition, $Z_{\mathrm{Ca}(Y)}\left(T, O_{P, Y}\right)=Z_{\mathrm{Ca}(Y)}\left(T, \widehat{O}_{P, Y}\right)$, where $\widehat{O}_{P, Y}$ is the completion of $O_{P, Y}$ with respect to the topology induced by its maximal ideal. If $\widehat{O}_{P, Y}$ is totally rational, then $Z_{\mathrm{Ca}(Y)}\left(T, \widehat{O}_{P, Y}\right)$ is completely determined by the semigroup of $\widehat{O}_{P, Y}$ (see [29, Lemma 5.4, Theorem 5.5]).

REMARK 11 . In the category of $\mathbb{F}_{q}$-algebraic varieties, [.] specializes to the counting rational points additive invariant $\#(\cdot)$. In addition, for a cylindric subset $X \subset \mathbb{P} \widetilde{\mathcal{O}}$ such that $X=\pi_{\underline{n}}^{-1}(Y)$ for a constructible subset $Y$ of $\mathbb{P} J \frac{n}{\widetilde{\mathcal{O}}}$, the only way to define the generalized Euler characteristic $\chi_{g}(X)$ of $X$ is by specializing $[\cdot]$ to the counting map \# $(\cdot)$ that gives the number of $\mathbb{F}_{q}$-rational points of a variety; that is,

$$
\chi_{g}(X)=\#(Y) \cdot q^{-\|\underline{n}+\underline{1}\|}
$$

(see, e.g., [11]). We denote by $\#\left(Z\left(T_{1}, \ldots, T_{d}, \mathcal{O}\right)\right)$ the rational function obtained by specializing $[\cdot]$ to \# $(\cdot)$. From a computational point of view, $\#\left(Z\left(T_{1}, \ldots, T_{d}, \mathcal{O}\right)\right)$ is obtained from $Z\left(T_{1}, \ldots, T_{d}, \mathcal{O}\right)$ by replacing $\mathbb{L}$ by $q$.

TheOREM 3. Let $k=\mathbb{F}_{q}$, and let $\mathcal{Z}\left(T_{1}, \ldots, T_{d}, U, S\right)$ be the universal zeta function for $S$. Let $Y$ be an algebraic curve defined over $k$, and let $\widehat{O}_{P, Y}$ be the completion of the local ring of $Y$ at a singular point $P$. Assume that $k$ is big enough for $Y$ and that $S=S\left(\widehat{O}_{P, Y}\right)$.

(1) For any $\mathcal{O}=\mathcal{O}_{\left(\mathbb{C}^{2}, 0\right)} /(f)$, with $f \in \mathcal{O}_{\left(\mathbb{C}^{2}, 0\right)}$ reduced, and $S=S(\mathcal{O})$,

$$
\begin{aligned}
Z_{\mathrm{Ca}(Y)}\left(q^{-1} T, q, \widehat{O}_{P, Y}\right) & =\#\left(Z\left(T_{1}, \ldots, T_{d}, \widehat{O}_{P, Y}\right)\right) \\
& =\left.\mathcal{Z}\left(T_{1}, \ldots, T_{d}, U, S\right)\right|_{T_{1}=\cdots=T_{d}=T .} .
\end{aligned}
$$

In particular, $Z_{\mathrm{Ca}(Y)}\left(q^{-1} T, q, \widehat{O}_{P, Y}\right)$ depends only on $S$. In addition, if $Y$ is a plane curve, then $Z_{\mathrm{Ca}(Y)}\left(q^{-1} T, q, \widehat{O}_{P, Y}\right)$ is a complete invariant of the equisingularity class of $\widehat{O}_{P, Y}$.

(2) For any $\mathcal{O}=\mathcal{O}_{\left(\mathbb{C}^{2}, 0\right)} /(f)$, with $f \in \mathcal{O}_{\left(\mathbb{C}^{2}, 0\right)}$, and $S=S(\mathcal{O})$,

$$
\left.Z_{\mathrm{Ca}(Y)}\left(q^{-1} T, q, \widehat{O}_{P, Y}\right)\right|_{q \rightarrow 1}=\varsigma_{f}(T)
$$


Proof. (1) Let $I=\left(z_{1}, \ldots, z_{d}\right) \widehat{O}_{P, Y} \subseteq \widehat{O}_{P, Y}$ be a principal ideal with

$$
\underline{n}=\left(v_{1}\left(z_{1}\right), \ldots, v_{d}\left(z_{d}\right)\right) .
$$

Since $\operatorname{dim}_{k}\left(\widehat{O}_{P, Y} / I\right)=\|\underline{n}\|$, and the number of ideals with "codimension $\underline{n} "$ is finite - this number is denoted as $\#\left(\mathcal{I}_{\underline{n}}\right)$ - we have

$$
Z_{\mathrm{Ca}(Y)}\left(q^{-1} T, q, \widehat{O}_{P, Y}\right)=\sum_{\underline{n} \in S\left(\widehat{O}_{P, Y}\right)} \#\left(\mathcal{I}_{\underline{n}}\right) q^{-\|\underline{n}\|} T^{\|\underline{n}\|} .
$$

On the other hand, by specializing [.] to \#(·) and by using the formula for $\left[\mathcal{I}_{\underline{n}}\right]$ given in Proposition 1, we obtain the explicit formula given for $\#\left(\mathcal{I}_{\underline{n}}\right)$ in [29, Lemma 5.4 ]; hence

$$
\begin{aligned}
Z_{\mathrm{Ca}(Y)}\left(q^{-1} T, q, \widehat{O}_{P, Y}\right) & =\#\left(Z\left(T_{1}, \ldots, T_{d}, \widehat{O}_{P, Y}\right)\right) \\
& =\left.Z\left(T, \ldots, T, \widehat{O}_{P, Y}\right)\right|_{\mathbb{L} \rightarrow q} \\
& =\left.\mathcal{Z}\left(T_{1}, \ldots, T_{d}, U, S\right)\right|_{\substack{T_{1}=\cdots=T_{d}=T \\
U=q}},
\end{aligned}
$$

where in the last equality we used Lemma 7.

(2) From (1) and by using Theorem 2, we have

$$
\begin{aligned}
\left.Z_{C a(Y)}\left(q^{-1} T, q, \widehat{O}_{P, Y}\right)\right|_{q \rightarrow 1} & =\left.\mathcal{Z}\left(T_{1}, \ldots, T_{d}, U, S\right)\right|_{\substack{T_{1}=\cdots=T_{d}=T \\
U=1}} \\
& =\varsigma_{f}(T)
\end{aligned}
$$

\section{$\S 8$. Functional equations}

In this section $k$ is a field of characteristic $p \geqslant 0$, and $\mathcal{O}$ is a Gorenstein and totally rational ring. Let $S=S(\mathcal{O})$. We give functional equations for $Z\left(T_{1}, \ldots, T_{d}, \mathcal{O}\right)$, for $\mathcal{Z}\left(T_{1}, \ldots, T_{d}, U, S\right)$, and for other Poincaré series.

Recall that for any $\underline{n} \in \mathbb{Z}^{d}$, we have $l(\underline{n})=\operatorname{dim}_{k}\left(\mathcal{O} / J_{\underline{n}}(\mathcal{O})\right)$, with $J_{\underline{n}}=$ $\{\underline{z} \in \mathcal{O} \mid \underline{v}(\underline{z}) \geqslant \underline{n}\}$ (see Section 5). In addition, we have

$$
l(\underline{n})=l\left(\underline{n}-\underline{e}_{i}\right)+\operatorname{dim}_{k}\left(J_{\underline{n}-\underline{e}_{i}}(\mathcal{O}) / J_{\underline{n}}(\mathcal{O})\right), \quad \text { for all } \underline{n} \in \mathbb{Z}^{d} .
$$

The following result can be found in [8, Theorem (3.6)]. 
Lemma 8. For any $\underline{n} \in \mathbb{Z}^{d}$ and any $i \in\{1, \ldots, d\}$, we have

$$
\operatorname{dim}_{k}\left(J_{\underline{n}}(\mathcal{O}) / J_{\underline{n}+\underline{e}_{i}}(\mathcal{O})\right)+\operatorname{dim}_{k}\left(J_{\underline{c}-\underline{n}-\underline{e}_{i}}(\mathcal{O}) / J_{\underline{c}-\underline{n}}(\mathcal{O})\right)=1 .
$$

The following result will be used in the proof of the functional equation.

LEMMA 9.

$$
l(\underline{c}-\underline{n})-l(\underline{n})=\delta-\|\underline{n}\|, \quad \underline{n} \in \mathbb{Z}^{d} .
$$

Proof. We use induction on $\|\underline{m}\|:=\sum_{i=1}^{d}\left|m_{i}\right|$, where $\underline{m}=\left(m_{1}, \ldots\right.$, $\left.m_{d}\right) \in \mathbb{Z}^{d}$. For $\|\underline{m}\|=0$ we have $\underline{m}=\underline{0}$. In this case, $l(\underline{0})=0, l(\underline{c})=\delta$, and the result is true. Assume, as an induction hypothesis, that the result is true for every $\underline{m} \in \mathbb{Z}^{d}$ with $\|\underline{m}\| \leqslant k$ for some $k \geqslant 1$. From the induction hypothesis, we have the following two formulas:

(i) if $0<\|\underline{m}\| \leqslant k$ and $m_{i} \geqslant 1$ for some $i \in\{1, \ldots, d\}$, then for $\underline{m}-\underline{e}_{i}$,

$$
l\left(\underline{c}-\left(\underline{m}-\underline{e}_{i}\right)\right)-l\left(\underline{m}-\underline{e}_{i}\right)=\delta-\left\|\underline{m}-\underline{e}_{i}\right\| ;
$$

(ii) if $0<\|\underline{m}\| \leqslant k$ and $m_{i} \leqslant 0$, then for some $i \in\{1, \ldots, d\}, m_{i}<0$. Then for $\underline{m}+\underline{e}_{i}$,

$$
l\left(\underline{c}-\left(\underline{m}+\underline{e}_{i}\right)\right)-l\left(\underline{m}+\underline{e}_{i}\right)=\delta-\left\|\underline{m}+\underline{e}_{i}\right\| .
$$

We now verify the validity of the result for $\|\underline{m}\|=k+1$. If $m_{i} \geqslant 1$ for some $i \in\{1, \ldots, d\}$, by applying (8.1),

$$
l(\underline{c}-\underline{m})-l(\underline{m})=l(\underline{c}-\underline{m})-l\left(\underline{m}-\underline{e}_{i}\right)-\operatorname{dim}_{k}\left(J_{\underline{m}-\underline{e}_{i}}(\mathcal{O}) / J_{\underline{m}}(\mathcal{O})\right),
$$

we now use Lemma 8 and (8.1) to get

$$
\begin{aligned}
l(\underline{c}-\underline{m})-l(\underline{m}) & =l(\underline{c}-\underline{m})-l\left(\underline{m}-\underline{e}_{i}\right)-\left(1-\operatorname{dim}_{k}\left(J_{\underline{c}-\underline{m}}(\mathcal{O}) / J_{\underline{c}-\underline{m}+\underline{e}_{i}}(\mathcal{O})\right)\right) \\
& =l(\underline{c}-\underline{m})+\operatorname{dim}_{k}\left(J_{\underline{c}-\underline{m}}(\mathcal{O}) / J_{\underline{c}-\underline{m}+\underline{e}_{i}}(\mathcal{O})\right)-l\left(\underline{m}-\underline{e}_{i}\right)-1 \\
& =l\left(\underline{c}-\left(\underline{m}-\underline{e}_{i}\right)\right)-l\left(\underline{m}-\underline{e}_{i}\right)-1 .
\end{aligned}
$$

Finally, by applying induction hypothesis (8.2), we get

$$
l(\underline{c}-\underline{m})-l(\underline{m})=\delta-\|\underline{m}\| .
$$

In the case in which $m_{i}<0$, for some $i \in\{1, \ldots, d\}$, we apply the previous reasoning and induction hypothesis (8.3) to get

$$
l(\underline{c}-\underline{m})-l(\underline{m})=\delta-\|\underline{m}\| .
$$


REMARK 12 . We note that $\mathcal{I}_{\underline{n}}=\emptyset$ whenever $\underline{n} \notin S$; thus, $\left[\mathcal{I}_{\underline{n}}\right]=0$ if $\underline{n} \notin S$. We can write $Z\left(T_{1}, \ldots, T_{d}, \mathcal{O}\right)$ as follows:

$$
Z\left(T_{1}, \ldots, T_{d}, \mathcal{O}\right)=\sum_{\underline{n} \in \mathbb{Z}^{d}}\left[\mathcal{I}_{\underline{n}}\right] \mathbb{L}^{-\|\underline{n}\|} T^{\underline{n}} .
$$

Theorem 4. Let $\mathcal{O}$ be a Gorenstein and totally rational ring. Assume that $\mathcal{J} \cong\left(G_{m}\right)^{d-1} \times\left(G_{a}\right)^{\delta-d+1}$; then

(1)

$$
Z\left(\mathbb{L} T_{1}, \ldots, \mathbb{L} T_{d}, \mathcal{O}\right)=\mathbb{L}^{\delta-d} \cdot T^{\underline{c}-1} \cdot \frac{\prod_{i=1}^{d}\left(1-\mathbb{L} T_{i}\right)}{\prod_{i=1}^{d}\left(T_{i}-1\right)} \cdot Z\left(T_{1}^{-1}, \ldots, T_{d}^{-1}, \mathcal{O}\right) ;
$$

$$
\mathcal{Z}\left(U T_{1}, \ldots, U T_{d}, U, S\right)=U^{\delta-d} \cdot T^{\underline{c}-1} \cdot \frac{\prod_{i=1}^{d}\left(1-U T_{i}\right)}{\prod_{i=1}^{d}\left(T_{i}-1\right)} \cdot \mathcal{Z}\left(T_{1}^{-1}, \ldots, T_{d}^{-1}, U, S\right)
$$

Proof. (1) We first note that

$$
\begin{aligned}
\left(\prod_{i=1}^{d}\left(T_{i}-1\right)\right) Z\left(\mathbb{L} T_{1}, \ldots, \mathbb{L} T_{d}, \mathcal{O}\right) & =\left(\prod_{i=1}^{d}\left(T_{i}-1\right)\right) \sum_{\underline{n} \in \mathbb{Z}^{d}}\left[\mathcal{I}_{\underline{n}}\right] T^{\underline{n}} \\
& =\sum_{\underline{n} \in \mathbb{Z}^{d}} \sum_{J \subseteq I_{0}}(-1)^{d-\# J}\left[\mathcal{I}_{\underline{n}}\right] T^{\underline{n}+\underline{1}_{J}} \\
& =\sum_{\underline{n} \in \mathbb{Z}^{d}} \sum_{J \subseteq I_{0}}(-1)^{d-\# J}\left[\mathcal{I}_{\underline{n}-\underline{1}_{J}}\right] T^{\underline{n}},
\end{aligned}
$$

where $I_{0}=\{1,2, \ldots, d\}$ and, for $J \subseteq I_{0}, \underline{1}_{J}$ is the element of $\mathbb{N}^{d}$ whose $i$ th component is equal to 1 or 0 , if $i \in J$ or if $i \notin J$, respectively. If $\underline{n}-\underline{1}_{J} \notin S$, then $\left[\mathcal{I}_{\underline{n}-\underline{1}_{J}}\right]=0$; if $\underline{n}-\underline{1}_{J} \in S$, then by applying Proposition 1 ,

$$
\begin{aligned}
& \left(\prod_{i=1}^{d}\left(T_{i}-1\right)\right) Z\left(\mathbb{L} T_{1}, \ldots, \mathbb{L} T_{d}, \mathcal{O}\right) \\
& \quad=\frac{\mathbb{L}}{\mathbb{L}-1} \sum_{\underline{n} \in \mathbb{Z}^{d}} \sum_{J \subseteq I_{0}}(-1)^{d-\# J} \sum_{I \subseteq I_{0}}(-1)^{\# I} \mathbb{L}^{\left\|\underline{n}-\underline{1}_{J}\right\|-l\left(\underline{n}+\underline{1}_{I}-\underline{1}_{J}\right)} T^{\underline{n}} .
\end{aligned}
$$

Taking into account that $\mathcal{O}$ is Gorenstein, that is, $\|\underline{c}\|=2 \delta$, and applying Lemma 9,

$$
l\left(\underline{n}+\underline{1}_{I}-\underline{1}_{J}\right)=\left\|\underline{n}+\underline{1}_{I}-\underline{1}_{J}\right\|+l\left(\underline{c}-\underline{n}-\underline{1}_{I}+\underline{1}_{J}\right)-\delta,
$$


and $\left(\prod_{i=1}^{d}\left(T_{i}-1\right)\right) Z\left(\mathbb{L} T_{1}, \ldots, \mathbb{L} T_{d}, \mathcal{O}\right)$ becomes

$$
\begin{aligned}
& \frac{\mathbb{L}}{\mathbb{L}-1} \sum_{\underline{n} \in \mathbb{Z}^{d}} \sum_{J \subseteq I_{0}}(-1)^{d-\# J} \sum_{I \subseteq I_{0}}(-1)^{\# I} \mathbb{L}^{\left\|\underline{n}-\underline{1}_{J}\right\|-\left\|\underline{n}+\underline{1}_{I}-\underline{1}_{J}\right\|-l\left(\underline{c}-\underline{n}-\underline{1}_{I}+\underline{1}_{J}\right)+\delta} T^{\underline{n}} \\
& =\frac{\mathbb{L}}{\mathbb{L}-1} \sum_{\underline{n} \in \mathbb{Z}^{d}} \sum_{I \subseteq I_{0}} \sum_{J \subseteq I_{0}}(-1)^{d-\# J+\# I} \mathbb{L}^{-\delta+\|\underline{n}\|} \mathbb{L}^{\left\|\underline{c}-\underline{n}-\underline{1}_{I}\right\|-l\left(\underline{c}-\underline{n}-\underline{1}_{I}+\underline{1}_{J}\right)} T^{\underline{n}} \\
& =\sum_{\underline{n} \in \mathbb{Z}^{d}} \sum_{I \subseteq I_{0}}(-1)^{d+\# I} \mathbb{L}^{-\delta+\|\underline{n}\|} \\
& \times\left(\frac{\mathbb{L}}{\mathbb{L}-1} \sum_{J \subseteq I_{0}}(-1)^{\# J} \mathbb{L}^{\left\|\underline{c}-\underline{n}-\underline{1}_{I}\right\|-l\left(\underline{c}-\underline{n}-\underline{1}_{I}+\underline{1}_{J}\right)}\right) T^{\underline{n}} \\
& =\sum_{\underline{n} \in \mathbb{Z}^{d}} \mathbb{L}^{-\delta+\|\underline{n}\|} \sum_{I \subseteq I_{0}}(-1)^{d-\# I}\left[\mathcal{I}_{\underline{c}-\underline{n}-\underline{1}_{I}}\right] T^{\underline{n}} \\
& =\sum_{\underline{n} \in \mathbb{Z}^{d}} \sum_{I \subseteq I_{0}}(-1)^{d-\# I}\left[\mathcal{I}_{\underline{c}-\underline{n}-\underline{1}_{I}}\right] \mathbb{L}^{-\delta+\|\underline{n}\|} T^{\underline{n}} \\
& =\sum_{\underline{n} \in \mathbb{Z}^{d}} \sum_{I \subseteq I_{0}}(-1)^{d-\# I}\left[\mathcal{I}_{\underline{c}-\underline{n}-\underline{1}_{I}}\right] \mathbb{L}^{\delta-\|\underline{c}-\underline{n}\|} T^{\underline{n}} \\
& =\sum_{\underline{m} \in \mathbb{Z}^{d}} \sum_{I \subseteq I_{0}}(-1)^{d-\# I}\left[\mathcal{I}_{\underline{m}-\underline{1}_{I}}\right] \mathbb{L}^{\delta-\|\underline{m}\|} T^{\underline{c}-\underline{m}} \\
& =\mathbb{L}^{\delta} T^{\underline{c}} \sum_{\underline{m} \in \mathbb{Z}^{d}} \sum_{I \subseteq I_{0}}(-1)^{d-\# I}\left[\mathcal{I}_{\underline{m}-\underline{1}_{I}}\right]\left(\mathbb{L} T_{1}\right)^{-m_{1}} \times \cdots \times\left(\mathbb{L} T_{d}\right)^{-m_{d}} \\
& =\mathbb{L}^{\delta} T^{\underline{c}}\left(\prod_{i=1}^{d}\left(\left(\mathbb{L} T_{i}\right)^{-1}-1\right)\right) \sum_{\underline{m} \in \mathbb{Z}^{d}}\left[\mathcal{I}_{\underline{m}}\right]\left(\mathbb{L} T_{1}\right)^{-m_{1}} \times \cdots \times\left(\mathbb{L} T_{d}\right)^{-m_{d}} \\
& =\mathbb{L}^{\delta-d} T^{\underline{c}-\underline{1}}\left(\prod_{i=1}^{d}\left(1-\mathbb{L} T_{i}\right)\right) Z\left(T_{1}^{-1}, \ldots, T_{d}^{-1}, \mathcal{O}\right) .
\end{aligned}
$$

(2) The functional equation for $\mathcal{Z}\left(T_{1}, \ldots, T_{d}, U, S\right)$ follows from the first part by Definition 8 and Theorem 1 .

It is worth mentioning that, since we have not shown that

$$
\sum_{\underline{n} \in \mathbb{Z}^{d}} \mathcal{I}_{\underline{n}}(U) U^{-\|\underline{n}\|} T^{\underline{n}}=\mathcal{Z}\left(T_{1}, \ldots, T_{d}, U, S\right),
$$


it is necessary to show first the functional equation for $Z\left(T_{1}, \ldots, T_{d}, \mathcal{O}\right)$.

Corollary 5. If $\prod_{i=1}^{d}\left(1-\mathbb{L}^{-1} T_{i}\right) Z\left(T_{1}, \ldots, T_{d}, \mathcal{O}\right)=M\left(T_{1}, \ldots, T_{d}, \mathcal{O}\right)$, with

$$
M\left(T_{1}, \ldots, T_{d}, \mathcal{O}\right)=\sum_{\underline{0} \leqslant \underline{i} \leqslant \underline{c}} a_{\underline{i}} T^{\underline{i}}
$$

then $(1) M\left(\mathbb{L} T_{1}, \ldots, \mathbb{L} T_{d}, \mathcal{O}\right)=\mathbb{L}^{\delta} T^{c} M\left(T_{1}^{-1}, \ldots, T_{d}^{-1}, \mathcal{O}\right) ;(2) a_{\underline{i}}=a_{\underline{c}-\underline{i}} \mathbb{L}^{\delta-\|\underline{i}\|}$, for $\underline{0} \leqslant \underline{i} \leqslant \underline{c}$. In particular, $a_{\underline{c}}=\mathbb{L}^{-\delta}$, since $a_{\underline{0}}=1$, and then the degree of $M\left(T_{1}, \ldots, T_{d}, \mathcal{O}\right)$ is $\|\underline{c}\|$.

REMARK 13. (1) By specialization, several functional equations can be obtained, among them,

$$
Z(\mathbb{L} T, \mathcal{O})=\mathbb{L}^{\delta-d} \cdot T^{\| \underline{c}-1} \| \cdot\left(\frac{1-\mathbb{L} T}{T-1}\right)^{d} \cdot Z\left(T^{-1}, \mathcal{O}\right) .
$$

(2) By Lemma 4 one also obtains the functional equations for the generalized Poincaré series (see also [19, Theorem 5.4.3]):

$$
P_{g}\left(\mathbb{L} T_{1}, \ldots, \mathbb{L} T_{d}, \mathcal{O}\right)=\mathbb{L}^{\delta-d} \cdot T^{\underline{c}-1} \cdot \frac{\prod_{i=1}^{d}\left(1-\mathbb{L} T_{i}\right)}{\prod_{i=1}^{d}\left(T_{i}-1\right)} \cdot P_{g}\left(T_{1}^{-1}, \ldots, T_{d}^{-1}, \mathcal{O}\right) .
$$

(3) Let $\mathcal{O}=\mathcal{O}_{\left(\mathbb{C}^{2}, 0\right)} /(f)$, where $f \in \mathcal{O}_{\left(\mathbb{C}^{2}, 0\right)}$ is reduced. Then

$$
\varsigma_{f}(T)=(-1)^{d} T^{\|\underline{\underline{c}}-\underline{1}\|} \cdot \varsigma_{f}\left(T^{-1}\right) .
$$

\section{§9. Examples}

ExAmple 9.1. Set $\mathcal{O}=\mathbb{C}\{\{x, y\}\} /\left(x^{3}-y^{2}\right)$, and set $\widetilde{\mathcal{O}}=\mathbb{C}\{\{t\}\} ;$ then

$$
\mathcal{O}=\left\{\sum_{i=0}^{\infty} a_{i} t^{i} \in \widetilde{\mathcal{O}} \mid a_{1}=0\right\}=\mathbb{C}+t^{2} \mathbb{C}\{\{t\}\}
$$

and

$$
\mathcal{O}^{\times}=\left\{\sum_{i=0}^{\infty} a_{i} t^{i} \in \widetilde{\mathcal{O}} \mid a_{0} \neq 0, a_{1}=0\right\} .
$$

The semigroup of values is the set $\{0\} \cup\{n \in \mathbb{N} \mid n \geqslant 2\}$, and $\left[\pi_{\underline{c}-\underline{1}}\left(\mathcal{O}^{\times}\right)\right]=$ $\left[\mathbb{C}^{\times} \times\{\right.$point $\left.\}\right]=\mathbb{L}-1$. The group $\mathcal{J}$ is isomorphic to $\{1+b t \mid b \in \mathbb{C}\}$, where the product is defined as $\left(1+b_{0} t\right)\left(1+b_{1} t\right)=1+\left(b_{0}+b_{1}\right) t$, and the identity is 1 . We now compute the zeta function of $Z(T, \mathcal{O})$. We first note that $\left[\mathcal{I}_{0}\right]=[\{$ point $\}]=1$. To compute $\left[\mathcal{I}_{k}\right]$ for $k \geqslant 2$, we fix a set of polynomial 
representatives $\{\mu\}$ of $\mathcal{J}$ in $\widetilde{\mathcal{O}}$. If $I=z \mathcal{O}, v(z)=k$, then $z=t^{k}(1+b t) v$, with $1+b t \in \mathcal{J}$ and $v \in \mathcal{O}^{\times}$. The correspondence $z \rightarrow 1+b t$ gives a bijection between $\mathcal{I}_{k}$ and $\mathcal{J}$, for $k \geqslant 2$; therefore $\left[\mathcal{I}_{k}\right]=\mathbb{L}$, for $k \geqslant 2$, and $Z(T, \mathcal{O})=$ $\left(1-\mathbb{L}^{-1} T+\mathbb{L}^{-1} T^{2}\right) /\left(1-\mathbb{L}^{-1} T\right)$. Note that each $I$ in $\mathcal{I}_{k}$ corresponds to a $\mu \in \mathcal{J}$ such that $t^{k} \mu \in \mathcal{O}$.

By specializing $[\cdot]$ to the Euler characteristic $\chi(\cdot)$, we obtain $\chi(Z(T, \mathcal{O}))=$ $\left(1-T+T^{2}\right) /(1-T)=\varsigma_{f}(T)$. By applying a theorem of A'Campo (see [1]), it is possible to verify that $\chi(Z(T, \mathcal{O}))$ is the zeta function of the monodromy at the origin of the mapping $f: \mathbb{C}^{2} \rightarrow \mathbb{C}$, where $f(x, y)=x^{3}-y^{2}$.

Let $\mathbb{F}_{q}$ be a finite field with $q$ elements. Let us consider the local ring $\mathcal{A}=\mathbb{F}_{q} \llbracket x, y \rrbracket /\left(x^{3}-y^{2}\right)$, which is totally rational over $\mathbb{F}_{q}$. Observe that $\delta=1$ and that $\mathcal{J} \cong\left(\mathbb{F}_{q},+, 0\right)$. By specializing $[\cdot]$ to $\#(\cdot)$, we get $\#(Z(T, \mathcal{O}))=$ $Z\left(q^{-1} T, \mathcal{A}\right)$, where $Z(T, \mathcal{A})=\left(1-T+q T^{2}\right) /(1-T)$ is the local factor of the zeta function $Z(\mathrm{Ca}(X), T)$ at the origin; here $X$ is the projective curve over $\mathbb{F}_{q}$ defined by $f(x, y)=x^{3}-y^{2} \in \mathbb{F}_{q}[x, y]$. Note that $\lim _{q \rightarrow 1} Z(T, \mathcal{A})=\varsigma_{f}(T)$ (see [29, Example 5.6]).

EXAmple 9.2. Set $\mathcal{O}=\mathbb{C}\{\{x, y\}\} /\left(y^{2}-x^{4}+x^{5}\right)$, and set $\widetilde{\mathcal{O}}=\mathbb{C}\left\{\left\{t_{1}\right\}\right\} \times$ $\mathbb{C}\left\{\left\{t_{2}\right\}\right\}$; then

$$
\mathcal{O}=\left\{\left(\sum_{i=0}^{\infty} a_{i, 1} t_{1}^{i}, \sum_{i=0}^{\infty} a_{i, 2} t_{2}^{i}\right) \in \widetilde{\mathcal{O}} \mid a_{0,1}=a_{0,2}, a_{1,1}=a_{1,2}\right\} .
$$

The conductor ideal is $\mathcal{F}=\left(t_{1}^{2}, t_{2}^{2}\right) \widetilde{\mathcal{O}}$, and the semigroup $S$ is equal to

$$
\{(0,0)\} \cup\{(1,1)\} \cup\left\{\left(k_{1}, k_{2}\right) \in \mathbb{N}^{2} \mid k_{1} \geqslant 2, k_{2} \geqslant 2\right\} .
$$

Note that $\left[\pi_{\underline{c}-1}\left(\mathcal{O}^{\times}\right)\right]=(\mathbb{L}-1) \mathbb{L}$. The group $\mathcal{J}$ is isomorphic to

$$
\left\{\left(a+b t_{1}, 1\right) \mid a \in \mathbb{C}^{\times}, b \in \mathbb{C}\right\},
$$

where the product is defined as

$$
\left(a_{0}+b_{0} t_{1}, 1\right)\left(a_{1}+b_{1} t_{1}, 1\right)=\left(a_{0} a_{1}+\left(a_{0} b_{1}+a_{1} b_{0}\right) t_{1}, 1\right) .
$$

Therefore $[\mathcal{J}]=(\mathbb{L}-1) \mathbb{L}$, and $\left[\mathcal{I}_{\underline{n}}\right]=[\mathcal{J}]$, for $\underline{n} \geqslant(2,2)$. To compute $\left[\mathcal{I}_{(1,1)}\right]$, we use the fact that each $I$ in $\mathcal{I}_{(1,1)}$ corresponds to a point of $\underline{\mu}=\left(a+b t_{1}\right.$, $1) \in \mathcal{J}$ such that $\left(t_{1}, t_{2}\right) \underline{\mu} \in \mathcal{O}$; thus we have to determine all the $a \in \mathbb{C}^{\times}$and $b \in \mathbb{C}$ such that

$$
\left(t_{1}, t_{2}\right)\left(a+b t_{1}, 1\right)=\left(a t_{1}+b t_{1}^{2}, t_{2}\right) \in \mathcal{O} .
$$


(Here the product is in $\widetilde{\mathcal{O}}$ and not in $\mathcal{J}$.) Then $a=1, b \in \mathbb{C}$; thus $\left[\mathcal{I}_{(1,1)}\right]=\mathbb{L}$, and $Z\left(T_{1}, T_{2}, \mathcal{O}\right)$ is equal to

$$
\frac{1-\mathbb{L}^{-1} T_{1}-\mathbb{L}^{-1} T_{2}+\left(\mathbb{L}^{-1}+\mathbb{L}^{-2}\right) T_{1} T_{2}-\mathbb{L}^{-2} T_{1} T_{2}^{2}-\mathbb{L}^{-2} T_{1}^{2} T_{2}+\mathbb{L}^{-2} T_{1}^{2} T_{2}^{2}}{\left(1-\mathbb{L}^{-1} T_{1}\right)\left(1-\mathbb{L}^{-1} T_{2}\right)} .
$$

By specializing [.] to $\chi(\cdot)$, we have $\chi(Z(T, \mathcal{O}))=1+T^{2}=\varsigma_{f}(T)$, which are the Alexander polynomial and the zeta function of the monodromy of the germ of mapping $f: \mathbb{C}^{2} \rightarrow \mathbb{C}:(x, y) \mapsto y^{2}-x^{4}+x^{5}$ at the origin.

Set the local ring $\mathcal{A}=\mathbb{F}_{q} \llbracket x, y \rrbracket /\left(y^{2}-x^{4}+x^{5}\right)$. Observe that $\delta=2$ and that $\mathcal{J} \cong\left(\left(\mathbb{F}_{q}\right)^{\times}, \cdot\right) \times\left(\mathbb{F}_{q},+, 0\right)$. By specializing $[\cdot]$ to $\#(\cdot)$, we obtain the equality $\#(Z(T, \mathcal{O}))=Z\left(q^{-1} T, \mathcal{A}\right)$, where $Z(T, \mathcal{A})=\left(1-2 T+(q+1) T^{2}-2 q T^{3}+\right.$ $\left.q^{2} T^{4}\right) /(1-T)^{2}$ is the local factor of the zeta function $Z(\mathrm{Ca}(X), T)$ at the origin; here $X$ is the projective curve over $\mathbb{F}_{q}$ defined by $f(x, y)=y^{2}-x^{4}+$ $x^{5} \in \mathbb{F}_{q}[x, y]$. Note that $\lim _{q \rightarrow 1} Z(T, \mathcal{A})=\varsigma_{f}(T)$.

ExAmple 9.3. Set $\mathcal{O}=\mathbb{C}\left\{\left\{t^{3}, t^{4}, t^{5}\right\}\right\}$, and set $\widetilde{\mathcal{O}}=\mathbb{C}\left\{\left\{t_{1}\right\}\right\}$. The embedding dimension of $\mathcal{O}$ is 3 . The group $\mathcal{J}$ is isomorphic to $\left\{1+a t+b t^{2} \mid a, b \in\right.$ $\mathbb{C}$ \}, where the product is defined as

$$
\left(1+a_{0} t+b_{0} t^{2}\right)\left(1+a_{1} t+b_{1} t^{2}\right)=1+\left(a_{0}+a_{1}\right) t+\left(b_{0}+b_{1}+a_{0} a_{1}\right) t^{2} .
$$

The zeta function of this ring is $Z(T, \mathcal{O})=\left(1-\mathbb{L}^{-1} T+\mathbb{L}^{-1} T^{3}\right) /\left(1-\mathbb{L}^{-1} T\right)$ and $\chi(Z(T, \mathcal{O}))=\left(1-T+T^{3}\right) /(1-T)$. This rational function should be "the monodromy zeta function of $\mathcal{O}$," but this cannot be explained from the point of view of A'Campo [1]. It seems that the connection between $\chi(Z(T, \mathcal{O}))$ and the "topology of $\mathcal{O}$ " is not completely understood.

Acknowledgment. We thank the referee for useful comments, which led to an improvement of this work.

\section{REFERENCES}

[1] N. A'Campo, La fonction zêta d'une monodromie, Comment. Math. Helv. 50 (1975), 233-248.

[2] Y. André, An introduction to motivic zeta functions of motives, preprint, arXiv:0812.3920v1 [math.AG].

[3] F. Baldassarri, C. Deninger, and N. Naumann, "A motivic version of Pellikaan's two variable zeta function," in Diophantine Geometry, CRM Series 4, Ed. Norm., Pisa, 2007, 35-43.

[4] A. Campillo, F. Delgado, and S. M. Gusein-Zade, On the monodromy of a plane curve singularity and the Poincaré series of its ring of functions, Funct. Anal. Appl. 33 (1999), 56-57. 
[5] A. Campillo, F. Delgado, and S. M. Gusein-Zade, The Alexander polynomial of a plane curve singularity and integrals with respect to the Euler characteristic, Internat. J. Math. 14 (2003), 47-54.

[6] A. Campillo, F. Delgado, and S. M. Gusein-Zade, The Alexander polynomial of a plane curve singularity via the ring of functions on it, Duke Math. J. 117 (2003), $125-156$.

[7] A. Campillo, F. Delgado, and S. M. Gusein-Zade, Multi-index filtrations and generalized Poincaré series, Monatsh. Math. 150 (2007), 193-209.

[8] A. Campillo, F. Delgado, and K. Kiyek, Gorenstein property and symmetry for onedimensional local Cohen-Macaulay rings, Manuscripta Math. 83 (1994), 405-423.

[9] F. Delgado de la Mata, The semigroup of values of a curve singularity with several branches, Manuscripta Math. 59 (1987), 347-374.

[10] F. Delgado de la Mata, Gorenstein curves and symmetry of the semigroup of values, Manuscripta Math. 61 (1988), 285-296.

[11] F. Delgado de la Mata and J.-J. Moyano-Fernández, On the relation between the generalized Poincaré series and the Stöhr zeta function, Proc. Amer. Math. Soc. 137 (2009), 51-59.

[12] J. Denef and F. Loeser, Caracteristiques de Euler-Poincaré, fonctions zeta locales et modifications analytiques, J. Amer. Math. Soc. 5 (1992), 705-720.

[13] J. Denef and F. Loeser, Germs of arcs on singular algebraic varieties and motivic integration, Invent. Math. 135 (1999), 201-232.

[14] J. Denef and F. Loeser, "On some rational generating series occurring in arithmetic geometry," in Geometric Aspects of Dwork Theory, Vol. I, II, de Gruyter, Berlin, 2004, 509-526.

[15] V. M. Galkin, Zeta-functions of certain one-dimensional rings, Izv. Akad. Nauk SSSR Ser. Mat. 37 (1973), 3-19.

[16] B. Green, Functional equations for zeta functions of non-Gorenstein orders in global fields, Manuscripta Math. 64 (1989), 485-502.

[17] M. Kapranov, The elliptic curve in the S-duality theory and Eisenstein series for Kac-Moody groups, preprint, arXiv:math/0001005 [math.AG].

[18] F. Loeser, Seattle Lectures on motivic integration, preprint, 2006.

[19] J.-J. Moyano-Fernández, Poincaré series associated with curves defined over perfect fields, Ph.D. dissertation, Universidad de Valladolid, Valladolid, Spain, 2008.

[20] M. Rosenlicht, Equivalence relations on algebraic curves, Ann. of Math. 56 (1952), 169-191.

[21] M. Rosenlicht, Generalized Jacobian varieties, Ann. of Math. 59 (1954), 505-530.

[22] J.-P. Serre, Algebraic Groups and Class Fields, Grad. Texts Math. 117, Springer, New York, 1988.

[23] K.-O. Stöhr, On the poles of regular differentials of singular curves, Bol. Soc. Brasil. Mat. (N.S.) 24 (1993), 105-136.

[24] K.-O. Stöhr, Local and global zeta functions of singular algebraic curves, J. Number Theory $\mathbf{7 1}$ (1998), 172-202.

[25] W. Veys, "Arc spaces, motivic integration and stringy invariants," in Singularity Theory and Its Applications (Sapporo, Japan, 2003), Adv. Stud. Pure Math. 43, Math. Soc. Japan, 2006, 529-572.

[26] R. Waldi, Wertehalbgruppe und Singularität einer ebenen algebroiden Kurve, Ph.D. dissertation, Universität Regensburg, Regensburg, Germany, 1972. 
[27] H. von Jürgen Herzog and E. Kunz, Der kanonische Modul eines Cohen-MacaulayRings. Seminar über die lokale Kohomologietheorie von Grothendieck, Universität Regensburg, Wintersemester 1970/1971, Lecture Notes in Math. 238, Springer, Berlin, 1971.

[28] O. Zariski, The Moduli Problem for Plane Branches, Univ. Lecture Ser. 39, Amer. Math. Soc., Providence, 2006.

[29] W. A. Zúñiga-Galindo, Zeta functions and Cartier divisors on singular curves over finite fields, Manuscripta Math. 94 (1997), 75-88.

[30] W. A. Zúñiga-Galindo, Zeta functions of singular curves over finite fields, Rev. Colombiana Mat. 31 (1997), 115-124.

[31] W. A. Zúñiga-Galindo, Zeta functions of singular algebraic curves over finite fields, Ph.D. dissertation, Instituto Nacional de Matemática Pure e Aplicada, Rio de Janeiro, Brazil, 1996.

J. J. Moyano-Fernández

Institut für Mathematik

Universität Osnabrück

Albrechtstrasse 28a

49076 Osnabrück

Deutschland

jmoyano@mathematik . uni-osnabrueck. de

W. A. Zúñiga-Galindo

Centro de Investigación y de Estudios Avanzados del Instituto Politécnico Nacional Departamento de Matemáticas, Avenida Instituto Politécnico Nacional 2508

Colonia San Pedro Zacatenco

México Distrito Federal 07360

México

wazuniga@math.cinvestav.edu.mx 